\title{
THE LONG RUN OUTCOMES AND GLOBAL DYNAMICS OF A DUOPOLY GAME WITH MISSPECIFIED DEMAND FUNCTIONS
}

\author{
GIAN-ITALO BISCHI \\ Istituto di Scienze Economiche \\ University of Urbino \\ via Saff n.42, I-61029 Urbino, Italy \\ bischi@econ.uniurb.it \\ CARL CHIARELLA \\ School of Finance and Economics \\ University of Technology Sydney \\ P.O. Box 123, Broadway, NSW 2007, Australia \\ carl.chiarella@uts.edu.au \\ MICHAEL KOPEL \\ Department of Managerial Economics and Industrial Organization \\ University of Technology \\ Theresianumgasse 27, A-1040 Vienna, Austria \\ kopel@ibab.tuwien.ac.at
}

\begin{abstract}
In this paper we study a model of a quantity-setting duopoly market where firms lack knowledge of the market demand. Using a misspecified demand function firms determine their profit-maximizing choices of their corresponding perceived market game. For illustrative purposes we assume that the (true) demand function is linear and that the reaction functions of the players are quadratic. We then investigate the global dynamics of this game and characterize the number of steady states and their welfare properties. We study the basins of attraction of these steady states and present situations in which global bifurcations of their basins occur when model parameters are varied. The economic significance of our result is to show that in situations where players choose their actions based on a misspecified model of the environment, additional self-confirming steady states may emerge, despite the fact that the Nash-equilibrium of the game under perfect knowledge is unique. As a consequence the long run outcome of the game and overall welfare is highly dependent upon initial conditions.
\end{abstract}

Keywords: Oligopoly games; bounded rationality; bifurcations; misspecified demand (function); self-confirming steady states.

\section{Introduction}

In the literature on learning and adaptive behavior in games, various attempts have been made to capture the incomplete knowledge of economic agents e.g., about the 
market demand or the production capacity of their rivals and to demonstrate the phenomena that may occur as a result of this lack of information. Scholars have tried to answer the following questions in this line of research (see Fudenberg and Levine (1998), Marimon (1997), or Kirman and Salmon (1995)): If we understand an equilibrium in a game as a steady state of some non-equilibrium process of adjustment and "learning", what happens if agents use an incorrect model of their environment? Does a reasonable adaptive process (e.g., based on the best response) converge to anything? If so, to what does it converge? Is the limit that can be observed when agents play their perceived games (close to) an equilibrium of the underlying true model? Is the observed situation consistent with the (limit) beliefs of the agents? Using a simple model that is a development of that of Léonard and Nishimura (1999), we demonstrate in this paper that in situations where players choose their actions based on a misspecified model of the environment, additional self-confirming steady states may emerge, despite the fact that the Nash-equilibrium of the game under perfect knowledge is unique. Furthermore the various steady states of the game may have quite complicated basins of attraction. As a consequence the long run outcome of the game and overall welfare is highly dependent upon initial conditions.

In an early study Kirman (1975) considers a simple duopoly model, where he assumes that the duopolists, through ignorance or inertia, are not aware that their demand depends on each other's action. Furthermore, he assumes that the players choose their action such that the expected profit of the next period is maximized and the duopolists update their estimates of the parameters of the (misspecified) perceived model. Within this simple framework, he shows that instead of converging to the "true" situation, the beliefs of the agents may drive the model towards some

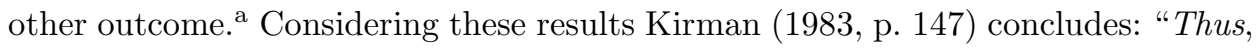
in a certain sense, rather than the agents learning about the model, the model learns from the agents, and the outcome is conditioned by their view of the world, mistaken or otherwise. Thus, we have a situation corresponding to the idea of 'self-fulfilling expectations' even though individuals are misinformed. It may well be argued that because agents are frequently less than perfectly informed, such situations may be commonplace." In addition to the result that agents are not able to learn the true equilibrium, it is also shown in Kirman $(1975,1983)$ that if convergence to the full information equilibrium fails, the process may become path-dependent, i.e., the particular equilibrium that can be observed depends on the starting conditions. Furthermore, Brousseau and Kirman (1993) find regions of stability as well as complicated dynamics in their simulations, whilst Kirman (1995) makes some remarks on basins of attraction. These observations are important, since they highlight the need to study the global dynamics of the economic game, in particular the characteristics of its possible long run outcomes and their respective basins of attraction.

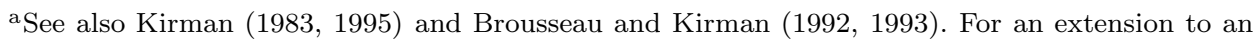
N-player market game and convergence results, see Gates et al. (1978, 1982). 
In our view this issue has been neglected in the literature ${ }^{\mathrm{b}}$ and a focus on this topic is one of the contributions of the present paper.

Léonard and Nishimura (1999) provide further evidence for the convergence of the economic game to a situation that is unrelated to any "real" equilibrium of the underlying market game. They consider a simple Cournot-duopoly model with decreasing reaction functions. Agents are aware of their rival and form expectations about the quantity choice of the other player. They show that if players (slightly) over- or underestimate the true demand, then an adaptive process based on the best replies converges towards a unique steady state that differs from the fullinformation (Nash-) equilibrium. They also demonstrate that this steady state may lose stability as the misspecification error (of one firm) becomes larger, and they provide an illustrative example where the system may converge to a period-2 cycle, with periodic points close to the unstable steady state. A similar result is obtained by Chiarella and Szidarovszky (2001) in an n-player continuous-time analog of the Léonard-Nishimura framework. They show that (under concavity assumptions) the resulting dynamic system converges towards a steady state, which in general differs from the full information equilibrium. They then introduce (continuously distributed) time lags in firms' reactions and demonstrate that in the modified model, limit cycles might be observed. Further results on the continuous-time analog of the Lèonard-Nishimura model can be found in Chiarella et al. (2002).

In this paper we extend the discrete time framework of Léonard and Nishimura (1999) in a number of ways. In Sec. 2, we set up a quite general formulation of a duopoly model where players lack knowledge of the market demand and where cost externalities between the firms are taken into account. For this model we derive the best reply dynamics. Our framework is more general in the sense that we do not restrict ourselves to a specific form of demand misspecification and as we demonstrate — the model introduced by Léonard and Nishimura (1999) is obtained as a special case. In Sec. 3 we recall recent criticism by several authors, who have stressed that the assumption of decreasing reaction functions is too restrictive (see e.g., Witteloostuijn and van Lier (1990), Bulow et al. (1985), Dana and Montrucchio, (1986)). One way to obtain reaction functions which are not monotonic is to allow for cost externalities between players, i.e., player $i$ 's total costs (e.g., for market development, product introduction, production and advertising) are influenced by the decisions of player $j$. As an illustrative example, we assume that demand is linear and players' reaction functions are quadratic (where the specific functional form of the reaction functions is obtained by suitably specifying the cost functions). We then go on to show that in this example for demand misspecification à la Léonard and Nishimura it is possible that new steady states

\footnotetext{
${ }^{\mathrm{b}}$ In a recent paper Schinkel et al. (2002) consider an oligopolistic price setting model where firms do not know the market demand but have demand conjectures instead. Beliefs are updated in a Bayesian way. They analyze the global dynamics and show that the particular equilibrium which is reached in the long run depends on the intial beliefs.
} 
are created, when (one or both) players over- or underestimate the demand. Such new states may even be "far away" from the full information Nash equilibrium in the strategy space and welfare in these perceived equilibria is lower than in the Nash equilibrium of the true game. For the case of identical players we provide a rigorous analysis of the existence and stability of the emerging steady states. For the case of heterogeneous players we give a numerical characterization of the various long run outcomes, of their basins of attraction and of the "global bifurcations" that change their topological structure as the parameters of the model are varied. In Sec. 4, we allow agents to react with some inertia and discuss how the results of Sec. 3 are thereby modified. Section 5 concludes.

\section{A Duopoly Model with Misspecified Demand}

We consider a discrete-time Cournot duopoly game, where firms $i=1,2$ offer homogeneous products on a common market. The demand function and the inverse demand are given by

$$
q=D(p), \quad \text { and } \quad p=D^{-1}(q) \equiv f(q)
$$

where $q=q_{1}+q_{2}$ denotes the industry output. However, firms do not know these relationships. Instead firm $i$ subjectively believes that the demand relationship is given by

$$
q=\tilde{D}_{i}(p), \quad(i=1,2)
$$

Accordingly, firm $i$ believes that the inverse demand function is given by

$$
p=\tilde{D}_{i}^{-1}(q) \equiv \tilde{f}_{i}(q), \quad(i=1,2)
$$

To keep the model as general as possible, we allow for externalities on the cost side. The total $\operatorname{costs} c_{i}$ of firm $i$ to offer a certain quantity $q_{i}$ on the market might also depend on the quantity produced and offered by the other player. Such externalities might, for example, be due to cost reductions for production inputs or the development of a "buying habit" for the product among consumers (Poston and Stewart (1979); Shaffer (1984)). We assume that players take this effect on their own costs into account. More precisely, at the beginning of period $t$ firm 1 chooses $q_{1}(t)$ such that the expected profit

$$
\Pi_{1}\left(q_{1}(t), q_{2}^{e, p r i o r}(t)\right)=q_{1}(t) \tilde{f}_{1}\left(q_{1}(t)+q_{2}^{e, p r i o r}(t)\right)-c_{1}\left(q_{1}(t), q_{2}^{e, p r i o r}(t)\right),
$$

is maximized, where the term $q_{2}^{e, p r i o r}(t)$ denotes firm 1's belief about the quantity chosen by its rival. Firm 1 uses its subjective demand relationship and considers the effect of the competitor's expected quantity $q_{2}^{e, p r i o r}(t)$ on its profit in order to 
choose the best response. The first order condition is

$$
\tilde{f}_{1}\left(q_{1}(t)+q_{2}^{e, p r i o r}(t)\right)+q_{1}(t)\left(\tilde{f}_{1}\right)^{\prime}\left(q_{1}(t)+q_{2}^{e, p r i o r}(t)\right)-\frac{\partial c_{1}\left(q_{1}(t), q_{2}^{e, p r i o r}(t)\right)}{\partial q_{1}} \leq 0,
$$

where for $q_{1}(t)>0$ the equality sign holds. Similarly for firm 2 we get the first order condition

$$
\tilde{f}_{2}\left(q_{1}^{e, p r i o r}(t)+q_{2}(t)\right)+q_{2}(t)\left(\tilde{f}_{2}\right)^{\prime}\left(q_{1}^{e, p r i o r}(t)+q_{2}(t)\right)-\frac{\partial c_{2}\left(q_{1}^{e, p r i o r}(t), q_{2}(t)\right)}{\partial q_{2}} \leq 0,
$$

where for $q_{2}(t)>0$ the equality sign holds.

When the firms determine their optimal quantities, they have the following information. At the time firm $i$ forms the expectation $q_{j}^{e, p r i o r}(t)$, it knows its own past quantities, however, it has not observed the price $p(t)$ yet (hence the superscript 'prior'). We also assume that it does not know the quantities offered by its rival in previous periods. Consequently, firms do not know the industry outputs and so cannot easily infer the true demand relationship. We are also assuming that the firms do not seek to learn about the demand function. We make this assumption not because we believe attempts to learn would not occur, but rather because we feel that the model studied in this paper can serve as a benchmark against which the impact of learning schemes can be assessed. For one attempt to introduce learning in the framework we develop here we refer the reader to Wenzelburger (2003).

We assume that the first order conditions for the two firms define reaction functions

$$
q_{1}(t)=\tilde{R}_{1}\left(q_{2}^{e, p r i o r}(t)\right) \quad \text { and } \quad q_{2}(t)=\tilde{R}_{2}\left(q_{1}^{e, p r i o r}(t)\right)
$$

Of course, these "best responses" are, in general, different from the reaction functions if firms would know perfectly the demand (henceforth referred to as reaction functions in the usual sense).

After shipping their selected quantities to the market, at the end of period $t$ both firms observe the market price $p(t)$. However, as mentioned above, they do not observe the industry output or the quantity supplied by its rival. Using the only publicly available signal $p(t)$ they update their belief on the rival's choice. This updated belief will be denoted as $q_{j}^{e, p o s t}(t)$. For firm 1 this belief $q_{2}^{e, p o s t}(t)$ is implicitly defined by the relationship

$$
p(t)=\tilde{f}_{1}\left(q_{1}(t)+q_{2}^{e, p o s t}(t)\right)
$$

or equivalently by

$$
q_{1}(t)+q_{2}^{e, p o s t}(t)=\tilde{D}_{1}(p(t))
$$


Since no further information becomes available between periods, following Léonard and Nishimura (1999), we assume that

$$
q_{2}^{e, p r i o r}(t+1)=q_{2}^{e, p o s t}(t),
$$

which via (7) yields

$$
p(t)=\tilde{f}_{1}\left(q_{1}(t)+q_{2}^{e, p r i o r}(t+1)\right)
$$

or equivalently

$$
q_{1}(t)+q_{2}^{e, p r i o r}(t+1)=\tilde{D}_{1}(p(t)) .
$$

Similarly, for firm 2 we have

$$
p(t)=\tilde{f}_{2}\left(q_{1}^{e, p r i o r}(t+1)+q_{2}(t)\right),
$$

or equivalently

$$
q_{1}^{e, p r i o r}(t+1)+q_{2}(t)=\tilde{D}_{2}(p(t)) .
$$

The dynamics of the duopoly with misspecified demand relationships can now be summarized as follows. Starting from initial expectations about the rival's output and given their subjective demand relationships, the duopolists choose their best replies according to (6). Subsequently, the price that clears the market is determined by the true (but unknown) market demand function (1). After observing the current price (but not the industry output), the firms use the relations (10) and (12) to update their beliefs on the rival's quantity. The expectation-feedback cycle then repeats itself. The dynamics of the system is captured by

$$
\begin{aligned}
q_{1}(t) & =\tilde{R}_{1}\left(q_{2}^{e, p r i o r}(t)\right), \\
q_{2}(t) & =\tilde{R}_{2}\left(q_{1}^{e, \text { prior }}(t)\right), \\
p(t) & =D^{-1}\left(q_{1}(t)+q_{2}(t)\right) \equiv f(q(t)), \\
q_{1}(t)+q_{2}^{e, p r i o r}(t+1) & =\tilde{D}_{1}(p(t)), \\
q_{1}^{e, p r i o r}(t+1)+q_{2}(t) & =\tilde{D}_{2}(p(t)) .
\end{aligned}
$$

Note that Eqs. (13)-(17) can be reduced to the system of difference equations

$$
\begin{aligned}
& q_{1}(t+1)=\tilde{R}_{1}\left[\tilde{D}_{1}\left(f\left(q_{1}(t)+q_{2}(t)\right)\right)-q_{1}(t)\right], \\
& q_{2}(t+1)=\tilde{R}_{2}\left[\tilde{D}_{2}\left(f\left(q_{1}(t)+q_{2}(t)\right)\right)-q_{2}(t)\right] .
\end{aligned}
$$

It is easy to see that in the special case analyzed by Léonard and Nishimura (1999),

$$
\tilde{D}_{1}(p)=\varepsilon D(p) \quad \text { and } \quad \tilde{D}_{2}(p)=D(p)
$$

i.e., where (only) one firm mistakenly over- or underestimates the actual demand by a factor of $\varepsilon$, the equations given in (18)-(19) can be written in a particular form. First, since firm 2 is assumed to know the true market demand function, 
$\tilde{f}_{2}=f$ and $\tilde{R}_{2}=R_{2}$ hold, where the latter denotes the reaction function in the usual sense. Hence, Eq. (19) becomes

$$
\begin{aligned}
q_{2}(t+1) & =\tilde{R}_{2}\left[\tilde{D}_{2}\left(f\left(q_{1}(t)+q_{2}(t)\right)\right)-q_{2}(t)\right] \\
& =R_{2}\left[D\left(f\left(q_{1}(t)+q_{2}(t)\right)\right)-q_{2}(t)\right] \\
& =R_{2}\left[q_{1}(t)\right]
\end{aligned}
$$

which coincides with Eq. (10) in Léonard and Nishimura (1999). Second, using $\tilde{D}_{1}(p)=\varepsilon D(p)$, Eq. (18) can be written as

$$
\begin{aligned}
q_{1}(t+1) & =\tilde{R}_{1}\left[\tilde{D}_{1}\left(f\left(q_{1}(t)+q_{2}(t)\right)\right)-q_{1}(t)\right] \\
& =\tilde{R}_{1}\left[(\varepsilon-1) q_{1}(t)+\varepsilon q_{2}(t)\right] .
\end{aligned}
$$

Note that this expression still involves the reaction function based on the misspecified market demand. However, it is straightforward to show that (22) can be expressed in terms of the reaction function in the usual sense. To see this, note that $\tilde{f}_{1}(q)=f\left(\varepsilon^{-1} q\right)$, and hence $\left(\tilde{f}_{1}\right)^{\prime}(q)=\varepsilon^{-1} f^{\prime}\left(\varepsilon^{-1} q\right)$. Assuming an interior solution, Eq. (4) yields

$$
f\left(\varepsilon^{-1} q_{1}(t)+\varepsilon^{-1} q_{2}^{e, p r i o r}(t)\right)+\varepsilon^{-1} q_{1}(t) f^{\prime}\left(\varepsilon^{-1} q_{1}(t)+\varepsilon^{-1} q_{2}^{e, p r i o r}(t)\right)=\frac{\partial c_{1}}{\partial q_{1}} .
$$

If firm 1 knew the true demand function, it would determine $q_{1}(t)$ according to the first order condition

$$
f\left(q_{1}(t)+q_{2}^{e, p r i o r}(t)\right)+q_{1}(t) f^{\prime}\left(q_{1}(t)+q_{2}^{e, p r i o r}(t)\right)=\frac{\partial c_{1}}{\partial q_{1}},
$$

which implicitly defines the relation $q_{1}(t)=R_{1}\left(q_{2}^{e, p r i o r}(t)\right)$. Hence, we can conclude that the first order condition (4) together with assumption (20) implicitly define the relation $\varepsilon^{-1} q_{1}(t)=R_{1}\left(\varepsilon^{-1} q_{2}^{e, p r i o r}(t)\right)$, where $R_{1}$ is the reaction function in the usual sense. Obviously, the relation between the reaction function $\tilde{R}_{1}$ and the reaction function in the usual sense is given by

$$
\tilde{R}_{1}\left(q_{2}^{e, p r i o r}(t)\right)=\varepsilon R_{1}\left(\varepsilon^{-1} q_{2}^{e, p r i o r}(t)\right) .
$$

Accordingly, Eq. (22) can be written as

$$
q_{1}(t+1)=\varepsilon R_{1}\left[\frac{\varepsilon-1}{\varepsilon} q_{1}(t)+q_{2}(t)\right],
$$

which coincides with Eq. (9) in Léonard and Nishimura (1999).

Note that, if both firms use misspecified demand functions of the type discussed above, i.e.,

$$
\tilde{D}_{i}(p)=\varepsilon_{i} D(p) \quad(i=1,2)
$$


then the dynamics of the market game is governed by

$$
\begin{aligned}
& q_{1}(t+1)=\varepsilon_{1} R_{1}\left[\frac{\varepsilon_{1}-1}{\varepsilon_{1}} q_{1}(t)+q_{2}(t)\right] \\
& q_{2}(t+1)=\varepsilon_{2} R_{2}\left[q_{1}(t)+\frac{\varepsilon_{2}-1}{\varepsilon_{2}} q_{2}(t)\right] .
\end{aligned}
$$

Obviously, if both players know the true demand $\left(\varepsilon_{1}=\varepsilon_{2}=1\right)$, then (25) reduces to the well-known Cournot tâtonnement dynamics given (in vector notation) by

$$
\left(q_{1}(t+1), q_{2}(t+1)\right)=\left(R_{1}\left(q_{2}(t)\right), R_{2}\left(q_{1}(t)\right)\right) .
$$

However, note that even if players over- or underestimate the demand by a certain factor $\left(\varepsilon_{i} \neq 1\right)$, the dynamics of the repeated duopoly game with subjective beliefs is still governed by equations only involving the reaction functions in the usual sense. This property makes the assumption of this particular kind of misspecification quite appealing for further analysis.

The dynamical system (26) has very particular properties, which mainly arise from the fact that the second iterate of the two-dimensional map is given by two decoupled one-dimensional maps, i.e., $\left(q_{1}(t+2), q_{2}(t+2)\right)=\left(R_{1} \circ R_{2}\left(q_{1}(t), R_{2} \circ\right.\right.$ $R_{1}\left(q_{2}(t)\right)$; for an analysis of such systems see Dana and Montrucchio (1986), Bischi et al. (2000), Canovas (2000) and Canovas and Linero (2001). However, these properties no longer hold if at least one player's demand function is misspecified. Hence, having $\varepsilon_{i} \neq 1$ causes a strong structural change in the dynamical system. In what follows we will focus on the difference equation system (25), and investigate the effects of mistaken beliefs on the long-run properties of the dynamical system, in particular on the existence and stability of steady states (or more complex attractors), and on the extent and topological structure of the basins of attractions of these steady states (or attractors).

\section{Multistability and Global Dynamics}

Usually, in standard textbook treatments of Cournot duopoly games, only the case of monotonic decreasing reaction functions is considered. Consequently, the Cournot tâtonnement process (26) can have at most one steady state, located at the intersection of the reaction curves. For example, Léonard and Nishimura (1999) study the system (25) with $\varepsilon_{1}=\varepsilon>0$ and $\varepsilon_{2}=1$ and their conditions on the demand and cost functions result in decreasing and identical reaction functions, $R_{1}(\cdot)=R_{2}(\cdot)=R(\cdot)$. There exists then a unique positive steady state of (25) for each $\varepsilon>0$. Using a particular kind of decreasing reaction function, they show that this steady state loses local stability and a stable cycle of period 2 becomes the unique attractor. 
However, as the early paper by Rand (1978) demonstrates, the array of dynamic phenomena is much broader if the assumption of monotonicity is relaxed and unimodal reaction functions are admitted. There are several reasons why reaction functions might be non-monotonic. ${ }^{\mathrm{c}}$ As mentioned before, each firm may benefit up to a certain point from the output of its rival due to a buying habit of the consumers (Poston and Stewart (1978)). Furthermore, as Bulow et al. (1985a, b) argue, firms' outputs might be strategic complements for small values of the individual quantities, an effect which can arise if firm $i$ tries to deter entry into the market by firm $j$. If the reaction functions are not strictly decreasing, then, although the Cournot tâtonnement (26) might have a unique Nash equilibrium, it may be the case that as the "error" parameters $\varepsilon_{i}$ are varied, the system (25) might have multiple steady states. In such a case, the creation of new steady states is entirely caused by the subjective beliefs of the agents. Then the role of the misspecification parameters $\varepsilon_{i}$ in the stability properties of the steady states is worth investigating since $\varepsilon_{i}$ serves as a measure of the accuracy of the subjective beliefs. If multistability occurs, i.e., if several coexisting attracting sets are present, the process becomes path-dependent, so that the long run outcome crucially depends on the initial condition. Hence, in such a situation it is important to gain some knowledge about the boundaries that separate the basins of attraction of the various coexisting attracting sets, and to ascertain their role in the occurrence of global bifurcations that change the topological structure of the basins. As a side remark, it can also be pointed out that, by tuning the "error" parameters $\varepsilon_{i}$, due to local bifurcations new kinds of attractors may appear, characterized by periodic or chaotic time paths in the long run.

In order to investigate these issues, we consider the following illustrative example. We let the inverse demand function be linear

$$
p=f\left(q_{1}, q_{2}\right)=a-b\left(q_{1}+q_{2}\right)
$$

and the reaction functions in the usual sense be given by ${ }^{\mathrm{d}}$

$$
R_{i}\left(q_{j}\right)=\mu_{i} q_{j}\left(1-q_{j}\right) \quad i, j=1,2 i \neq j
$$

where the quantities $\left(q_{1}, q_{2}\right)$ have to be chosen in the strategy space $[0,1]^{2}$ and $\mu_{i} \in[0,4]$. If both players' misspecification of demand is given by $\tilde{D}_{i}(p)=\varepsilon_{i} D(p)$, then the dynamics of our market game is given by

$$
\left(q_{1}(t+1), q_{2}(t+1)\right)=\tilde{T}\left(q_{1}(t), q_{2}(t)\right)
$$

\footnotetext{
${ }^{\mathrm{c}}$ Further arguments can be found in Shaffer (1984), Dana and Montrucchio (1986), Van Witteloostuijn and Van Lier (1990) and Puu (1991).

${ }^{\mathrm{d}}$ Unimodal reaction functions can be derived e.g., if demand is iso-elastic (see Bulow et al. (1985a, b) and Puu (1991)). The more specific form of quadratic reaction functions are obtained by choosing the cost functions with externalities as $c_{i}\left(q_{1}, q_{2}\right)=a q_{i}-b q_{i} q_{j}\left(1+2 \mu_{i}\right)+2 b \mu_{i} q_{i} q_{j}^{2}, i, j=$ $1,2, i \neq j$ (see Kopel (1996)), where the parameters $\mu_{i}$ measure the intensity of the externality.
} 
where the map $\tilde{T}$ is defined by

$$
\tilde{T}:\left\{\begin{array}{l}
q_{1}(t+1)=\mu_{1}\left[\varepsilon_{1} q_{2}(t)\left(1-q_{2}(t)\right)+\left(\varepsilon_{1}-1\right) q_{1}(t)\left(1-\frac{\varepsilon_{1}-1}{\varepsilon_{1}} q_{1}(t)-2 q_{2}(t)\right)\right], \\
q_{2}(t+1)=\mu_{2}\left[\varepsilon_{2} q_{1}(t)\left(1-q_{1}(t)\right)+\left(\varepsilon_{2}-1\right) q_{2}(t)\left(1-\frac{\varepsilon_{2}-1}{\varepsilon_{2}} q_{2}(t)-2 q_{1}(t)\right)\right] .
\end{array}\right.
$$

Observe that if both firms know the true demand function so that, $\varepsilon_{1}=\varepsilon_{2}=1$, then (29) reduces to the Cournot best reply dynamics given by

$$
T:\left\{\begin{array}{l}
q_{1}(t+1)=\mu_{1} q_{2}(t)\left(1-q_{2}(t)\right), \\
q_{2}(t+1)=\mu_{2} q_{1}(t)\left(1-q_{1}(t)\right) .
\end{array}\right.
$$

This useful benchmark case has already been studied by Kopel (1996) and Bischi et al. (2000).

\subsection{Multiple steady states induced by demand misspecification}

The fixed points of the map (29) are the real solutions of the algebraic system $\tilde{T}\left(q_{1}, q_{2}\right)=\left(q_{1}, q_{2}\right)$, which reduces to

$$
\begin{aligned}
& \mu_{1}\left(\varepsilon_{1}-1\right)^{2} q_{1}^{2}+2 \mu_{1} \varepsilon_{1}\left(\varepsilon_{1}-1\right) q_{1} q_{2}+\mu_{1} \varepsilon_{1}^{2} q_{2}^{2} \\
& \quad+\varepsilon_{1}\left(1-\mu_{1}\left(\varepsilon_{1}-1\right)\right) q_{1}-\mu_{1} \varepsilon_{1}^{2} q_{2}=0,
\end{aligned}
$$

and

$$
\begin{aligned}
& \mu_{2}\left(\varepsilon_{2}-1\right)^{2} q_{2}^{2}+2 \mu_{2} \varepsilon_{2}\left(\varepsilon_{2}-1\right) q_{1} q_{2}+\mu_{2} \varepsilon_{2}^{2} q_{1}^{2} \\
& \quad+\varepsilon_{2}\left(1-\mu_{2}\left(\varepsilon_{2}-1\right)\right) q_{2}-\mu_{2} \varepsilon_{2}^{2} q_{1}=0 .
\end{aligned}
$$

These equations represent two parabolas in the $\left(q_{1}, q_{2}\right)$-plane, denoted by $\mathcal{P}_{1}$ and $\mathcal{P}_{2}$, whose symmetry lines are given by the equations

$$
q_{2}=\frac{1-\varepsilon_{1}}{\varepsilon_{1}} q_{1}-\frac{1}{2} \frac{\left(\varepsilon_{1}-1\right)\left(1-\mu_{1}\left(\varepsilon_{1}-1\right)\right)-\mu_{1} \varepsilon_{1}^{2}}{\mu_{1}\left(\left(\varepsilon_{1}-1\right)^{2}+\varepsilon_{1}^{2}\right)},
$$

and

$$
q_{1}=\frac{1-\varepsilon_{2}}{\varepsilon_{2}} q_{2}-\frac{1}{2} \frac{\left(\varepsilon_{2}-1\right)\left(1-\mu_{2}\left(\varepsilon_{2}-1\right)\right)-\mu_{2} \varepsilon_{2}^{2}}{\mu_{2}\left(\left(\varepsilon_{2}-1\right)^{2}+\varepsilon_{2}^{2}\right)},
$$

respectively. Both parabolas pass through the origin $(0,0)$, the parabola $\mathcal{P}_{1}$ also intersects the vertical axis in the point $(0,1)$ and the parabola $\mathcal{P}_{2}$ intersects the horizontal axis in the point $(1,0)$. So, besides the trivial fixed point $E_{0}=(0,0)$, we may have one or three positive steady states, located at the intersections of the two parabolas and obtained as the real solutions of a cubic equation.

If $\varepsilon_{1}=1$, then the parabola $\mathcal{P}_{1}$ assumes the simpler expression $q_{1}=\mu_{1} q_{2}\left(1-q_{2}\right)$, has horizontal symmetry axis $q_{2}=1 / 2$, and coincides with the reaction curve $R_{1}$. Analogously, if $\varepsilon_{2}=1$, then the parabola $\mathcal{P}_{2}$ assumes the simpler expression 
$q_{2}=\mu_{2} q_{1}\left(1-q_{1}\right)$, has the vertical symmetry axis $q_{1}=1 / 2$, and coincides with the reaction curve $R_{2}$ (Fig. 1(a)). The intersection point (denoted as $N E$ in Fig. 1(a)) represents the Nash equilibrium of the game. As the parameters $\mu_{1}$ and/or $\mu_{2}$ are increased in the "true" game, two further positive (Nash-)equilibria may appear, which can be easily obtained as the solutions of a cubic equation. Accordingly, an equilibrium selection problem arises in the original game and the basins of these equilibria can be used to study the global properties (see Bischi and Kopel, 2001). On the other hand, if $\varepsilon_{i} \neq 1$ then the parabolas $\mathcal{P}_{1}$ and $\mathcal{P}_{2}$ no longer coincide with the reaction curves $R_{1}$ and $R_{2}$ and intersection points no longer correspond to Nash equilibria of the "true" game, but to equilibria of the "perceived" game. Setting $\varepsilon_{i} \neq 1$ has the effect of changing the slope of the symmetry axis of the corresponding parabola $\mathcal{P}_{i}$, which also become more (less) peaked as $\varepsilon_{i}$ increases (decreases). This may cause a higher or lower number of fixed points than Nash equilibria of the true game. Furthermore, some of these fixed points might be located "far away" from the Nash equilibrium in the strategy space. The qualitative representation in Fig. 1 captures the basic mechanism for the emergence of several perceived equilibria when one or both error parameters $\varepsilon_{i}$ are varied. If $\varepsilon_{1}=\varepsilon_{2}=1$ there is a unique positive Nash equilibrium given by the intersection point of the reaction curves. Figure 1(b) illustrates the possible change in the number and location of equilibria in the perceived game $\left(\varepsilon_{1} \neq 1, \varepsilon_{2} \neq 1\right)$. In the situation depicted here there exist 3 intersections of the two parabolas $\mathcal{P}_{1}$ and $\mathcal{P}_{2}$ in the interior of the unit square and, accordingly, 3 potential long run outcomes of the game (depending on the stability properties). If, for example, it turns out that $E_{S}$ and $E_{1}$ are locally asymptotically stable (cf. Fig. 6(b)), then adjustment and "learning" might guide the players to the equilibrium $E_{S}$ and, therefore, close to the Nash equilibrium of the true game. However, players might in the long run also end up in a situation represented by $E_{1}$ and, hence, far away from the Nash equilibrium of the original game. ${ }^{\mathrm{e}}$ In order to make this informal statement more precise, we will compare the welfare properties of the (unique and locally stable) Nash equilibrium and the locally stable perceived equilibria in what follows. The expressions for the profits $\Pi_{i}\left(q_{1}, q_{2}\right)$ of the players are given at the beginning of this section. The consumer surplus in the case of a linear inverse demand curve can be calculated by the expression $C S\left(q_{1}, q_{2}\right)=b\left(q_{1}+q_{2}\right)^{2}$. The welfare in a corresponding pair of quantities is then given by the sum of these expressions $W\left(q_{1}, q_{2}\right)=\Pi_{1}\left(q_{1}, q_{2}\right)+\Pi_{2}\left(q_{1}, q_{2}\right)+C S\left(q_{1}, q_{2}\right)$. Note that the demand parameter $b$ appears in all three expressions as a scaling factor. In the subsequent comparison we will neglect it as we are only interested in the ranking of equilibria.

A rigorous analysis of the properties of the difference equation system (25) even with quadratic reaction functions - is a challenging task. Therefore, we start (in Sec. 3.2) with the case of identical players, $\mu_{1}=\mu_{2}$ and $\varepsilon_{1}=\varepsilon_{2}$, i.e., players

eAlthough we only illustrate this phenomenon for the case of quadratic reaction functions, we may speculate that such situations are quite common when duopoly games with unimodal reaction functions and misspecified demand functions of the form $\tilde{D}_{i}(p)=\varepsilon_{i} D(p)$ are considered. 

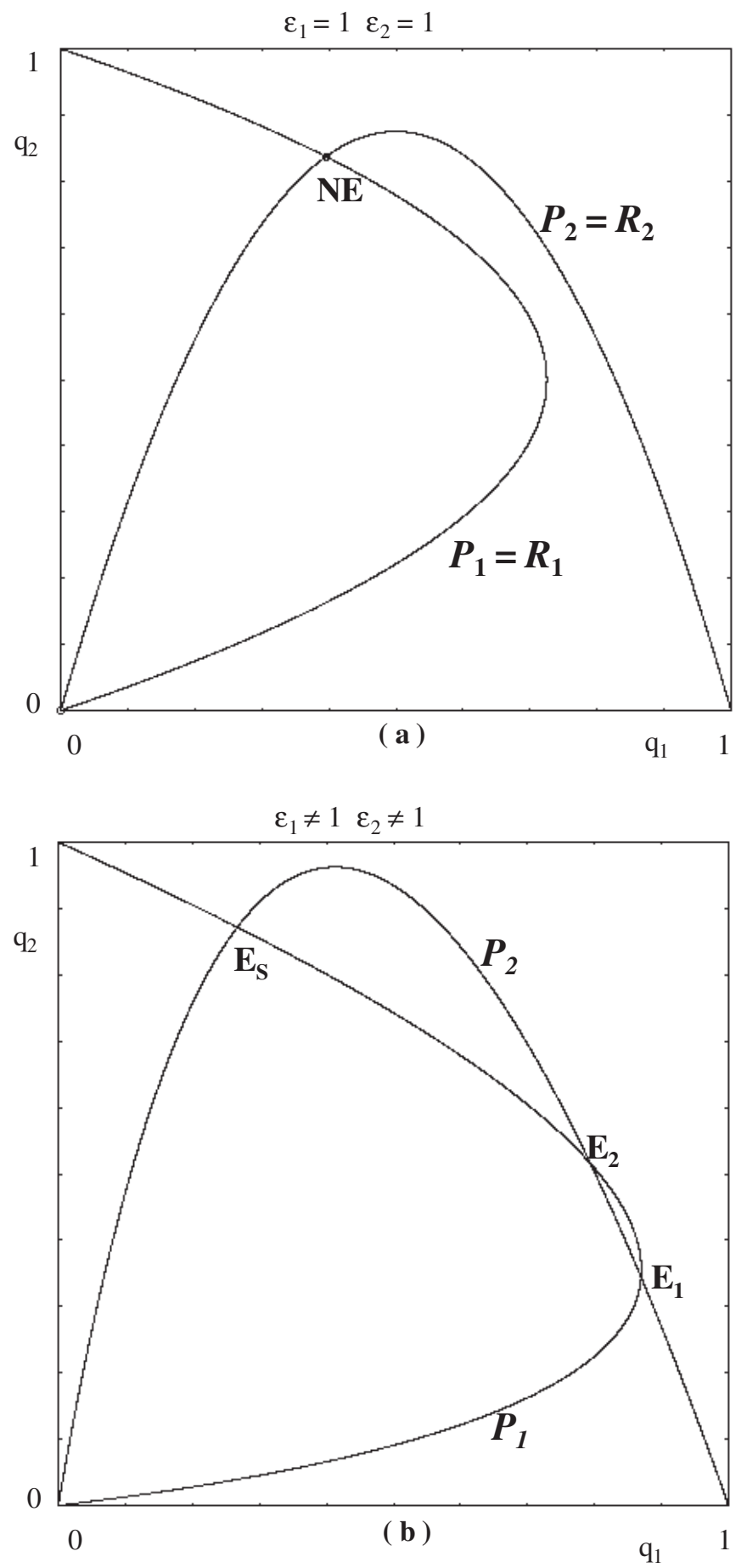

Fig. 1. Occurrence of multiple steady states due to misspecified demand (a) Reaction functions and the unique Nash equilibrium NE (b) Subjective reaction functions, and multiple steady states, obtained by increasing $\varepsilon_{i}$. 
are homogeneous with respect to their reaction functions and their accuracy of the subjective beliefs. Subsequently, on the basis of the analytic results obtained for the symmetric model, we move to the case of heterogeneous players (in Sec. 3.3). The heterogeneity may arise either with respect to their reaction functions, i.e., $\mu_{1} \neq \mu_{2}$, or their demand misspecification, i.e., $\varepsilon_{1} \neq \varepsilon_{2}$. On the basis of numerical simulations we will investigate local and global bifurcations that determine the number and the kind of possible long run outcomes as well as the structure of their basins of attraction. A welfare comparison will complement this analysis.

\subsection{The case of homogeneous players}

In this section we consider the case of homogeneous players, characterized by identical parameters

$$
\mu_{1}=\mu_{2}=\mu ; \quad \varepsilon_{1}=\varepsilon_{2}=\varepsilon .
$$

In Subsec. 3.2.1 we analyze the map that occurs in this case and the steady states to which it can give rise. In Subsec. 3.2 .2 we carry out a stability analysis, referring to results for the standard logistic map.

\subsubsection{The map and its steady states}

Under the assumption (32) the map (29) assumes the symmetric form

$$
\tilde{T}_{s}:\left\{\begin{array}{l}
q_{1}(t+1)=\mu\left[\varepsilon q_{2}(t)\left(1-q_{2}(t)\right)+(\varepsilon-1) q_{1}(t)\left(1-\frac{\varepsilon-1}{\varepsilon} q_{1}(t)-2 q_{2}(t)\right)\right], \\
q_{2}(t+1)=\mu\left[\varepsilon q_{1}(t)\left(1-q_{1}(t)\right)+(\varepsilon-1) q_{2}(t)\left(1-\frac{\varepsilon-1}{\varepsilon} q_{2}(t)-2 q_{1}(t)\right)\right],
\end{array}\right.
$$

where 'symmetry' refers to the fact that the map remains the same if the variables $q_{1}$ and $q_{2}$ are swapped. More formally, $\tilde{T}_{s} \circ S=S \circ \tilde{T}_{s}$, where $S:\left(q_{1}, q_{2}\right) \rightarrow\left(q_{2}, q_{1}\right)$ is the reflection through the diagonal $\Delta=\{(q, q), q \in \mathbb{R}\}$. This symmetry property implies that the diagonal $\Delta$ is a trapping subspace for the map $\tilde{T}_{s}$, i.e., $\tilde{T}_{s}(\Delta) \subseteq \Delta$. In other words, in a deterministic framework, identical players who choose the same initial quantities $q_{1}(0)=q_{2}(0)$, behave identically over time, i.e., $q_{1}(t)=q_{2}(t)$ for each $t \geq 0$. These trajectories along $\Delta$ are governed by a one-dimensional map which is obtained by restricting $\tilde{T}_{s}$ to $\Delta,\left.\tilde{T}_{s}\right|_{\Delta}: \Delta \rightarrow \Delta$, where the map $\left.\tilde{T}_{s}\right|_{\Delta}$ results from setting $q_{1}=q_{2}=q$ in Eq. (33) and is thus given by

$$
q(t+1)=\mu(2 \varepsilon-1) q(t)-\frac{\mu}{\varepsilon}(2 \varepsilon-1)^{2} q(t)^{2} .
$$

This map may be interpreted as a one-dimensional model of a "representative firm". ${ }^{\mathrm{f}}$ It is conjugate to the standard logistic map $z(t+1)=a z(t)(1-z(t))$, with

$$
a=\mu(2 \varepsilon-1)
$$

${ }^{\mathrm{f}}$ This point of view is proposed in Bischi et al. (1999) and Kopel et al. (2000). 
by the linear transformation $q=\frac{\varepsilon}{2 \varepsilon-1} z$. Using well-known dynamic properties of the logistic map we will study the properties of the two-dimensional map $\tilde{T}_{s}$.

Under assumption (32) the computation of the fixed points, i.e., real and positive solutions of $(34)$, becomes straightforward. In fact, apart from the trivial solution $E_{0}=(0,0)$, there is another solution given by

$$
E_{S}=\left(q_{S}, q_{S}\right) \in \Delta, \quad q_{S}=\frac{\varepsilon}{2 \varepsilon-1}\left(1-\frac{1}{\mu(2 \varepsilon-1)}\right),
$$

which represents a positive (symmetric) steady state provided that $(2 \varepsilon-1) \mu>1$. Two further solutions exist if $\Psi(\mu, \varepsilon)=(2 \varepsilon-1)(\mu+1)(\mu(2 \varepsilon-1)-(2 \varepsilon+1)) \geq 0$. They are located in symmetric positions with respect to the diagonal $\Delta$ and are given by

$$
E_{1}=\left(\bar{q}_{1}, \bar{q}_{2}\right) \quad \text { and } \quad E_{2}=\left(\bar{q}_{2}, \bar{q}_{1}\right)
$$

with

$$
\bar{q}_{1}=\varepsilon \frac{\mu+1+\sqrt{\Psi}}{2 \mu(2 \varepsilon-1)} \quad \text { and } \quad \bar{q}_{2}=\varepsilon \frac{\mu+1-\sqrt{\Psi}}{2 \mu(2 \varepsilon-1)} .
$$

We will restrict our analysis to situations where $\varepsilon>0.5$ holds, since $0<\varepsilon<0.5$ would represent an unreasonably strong underestimation of the demand. Hence, the condition for the existence of the two further steady states $E_{1}$ and $E_{2}$ can be written as

$$
\mu \geq \mu_{P} \equiv \frac{2 \varepsilon+1}{2 \varepsilon-1}
$$

or equivalently

$$
\varepsilon \geq \varepsilon_{P} \equiv \frac{\mu+1}{2(\mu-1)},
$$

where $\mu_{P}$ (or $\left.\varepsilon_{P}\right)$ plays the role of a critical bifurcation value. Accordingly, we can state that, for a given $\mu>1$, two steady states (close to $E_{S}$ in the homogeneous case that we are considering here) can be created, as the "misspecification" parameter $\varepsilon$ is increased across the bifurcation value $\varepsilon_{P}$.

\subsubsection{Stability analysis}

In this subsection we study the stability properties (in the $\mu-\epsilon$ parameter space) of the fixed points $E_{S}, E_{1}$ and $E_{2}$ of the map $\tilde{T}_{s}$. Recall that the map (34) is topologically conjugate to the standard logistic map $z(t+1)=a z(t)(1-z(t))$, where we know that the fixed point becomes unstable at $a=3$ and a stable twocycle emerges. For higher values of $a$ stable cycles of even higher period can be observed. Therefore, from (34) together with (35), we can deduce that increasing the parameters $\varepsilon$ and/or $\mu$ may lead to the creation of periodic cycles along the 
diagonal $\Delta$. In fact, the period doubling bifurcation of $E_{S}$, at which a cycle of period 2 is created along $\Delta$, occurs at

$$
\mu=\mu_{F} \equiv \frac{3}{2 \varepsilon-1} \text { or equivalently, } \varepsilon=\varepsilon_{F} \equiv \frac{\mu+3}{2 \mu} .
$$

The analysis of the eigenvalues of the Jacobian matrix of the symmetric twodimensional map $\tilde{T}_{s}$, outlined in Appendix A, allows us to give a complete description of the stability regions of the equilibria in the parameter space, i.e., we can obtain the analytic expressions of the curves that constitute the boundaries of such regions. The results of our stability analysis are summarized in Fig. 2, where the stability domain of $E_{S}$ in the space of the parameters $\varepsilon$ and $\mu$ is represented by the shaded region in Fig. 2(a), and the stability domain of the steady states $E_{1}$ and $E_{2}$ is represented by the shaded region in Fig. 2(b). Notice first that if players would know the true demand function $(\varepsilon=1)$, then for $1<\mu<3$ there is a unique, globally stable Nash equilibrium of the game, namely $N E=(1-1 / \mu, 1-1 / \mu)$ which in this case coincides with $E_{S}$. On the other hand, if players overestimate the demand $\left(\varepsilon_{F}<\varepsilon<\varepsilon_{P}\right)$, then cyclic (see also Léonard and Nishimura 1999) or even more complex behavior along the diagonal would be observed. ${ }^{g}$ Notice that if $1<\mu<3$, then $\varepsilon_{F}<\varepsilon_{P}$, so the two steady states $E_{i}, i=1,2$, are unstable (saddle points) when they are created at $\varepsilon=\varepsilon_{P}$. The two curves $\varepsilon=\varepsilon_{H}$ and $\varepsilon=\varepsilon_{F E_{i}}$, represented in Fig. 2(b) together with the curve $\varepsilon=\varepsilon_{P}$, bound a region in the $\mu-\epsilon$ parameter space where the steady states $E_{1}$ and $E_{2}$ are locally stable (these curves follow from the stability conditions given in the Appendix A) and coexist with the more complex attractor along the diagonal. So, summarizing the results of our stability analysis, we can say the following: there are situations where a unique and globally stable Nash equilibrium exists in the true game. However, agents playing a perceived game in which they overestimate the true demand, either adjust their actions towards $E_{1}$ or $E_{2}$ or keep on changing actions and beliefs from period to period.

Which of the long run outcomes will be observed obviously depends on the initial choices. In Fig. 3 we show the basins of attraction of the two stable equilibria $E_{1}$ and $E_{2}$ of the perceived game, and a coexisting two-cycle, obtained for $\mu=2.9$ and $\varepsilon=1.08$ (the two steady states are locally stable for an overestimation of demand between $3.6 \%$ and $18.6 \%$ ). We stress that for $\mu=2.9$ and $\varepsilon=1$, i.e., in the case of players who know the true demand function, a unique (Nash) equilibrium exists (it is represented by a small cross in Fig. 3) which is globally stable,

gIf $\varepsilon_{1}=\varepsilon_{2}$, then the stable cycle belongs to the invariant diagonal $\Delta$. The long-run dynamics along $\Delta$ are characterized by the period-doubling route to chaos as $\varepsilon_{1}$ and $\varepsilon_{2}$ are increased. For example, with $\mu_{1}=\mu_{2}=2.9$ and $\varepsilon_{1}=\varepsilon_{2}=1.1$, the stable cycle $C_{2}$ is replaced by a stable cycle of period 4 , and a further increase leads to a chaotic attractor along $\Delta$. If players are heterogeneous with slightly different values of $\varepsilon_{i}$, the chaotic attractor persists even if it is no longer trapped inside the diagonal; it becomes a two-dimensional chaotic area. 

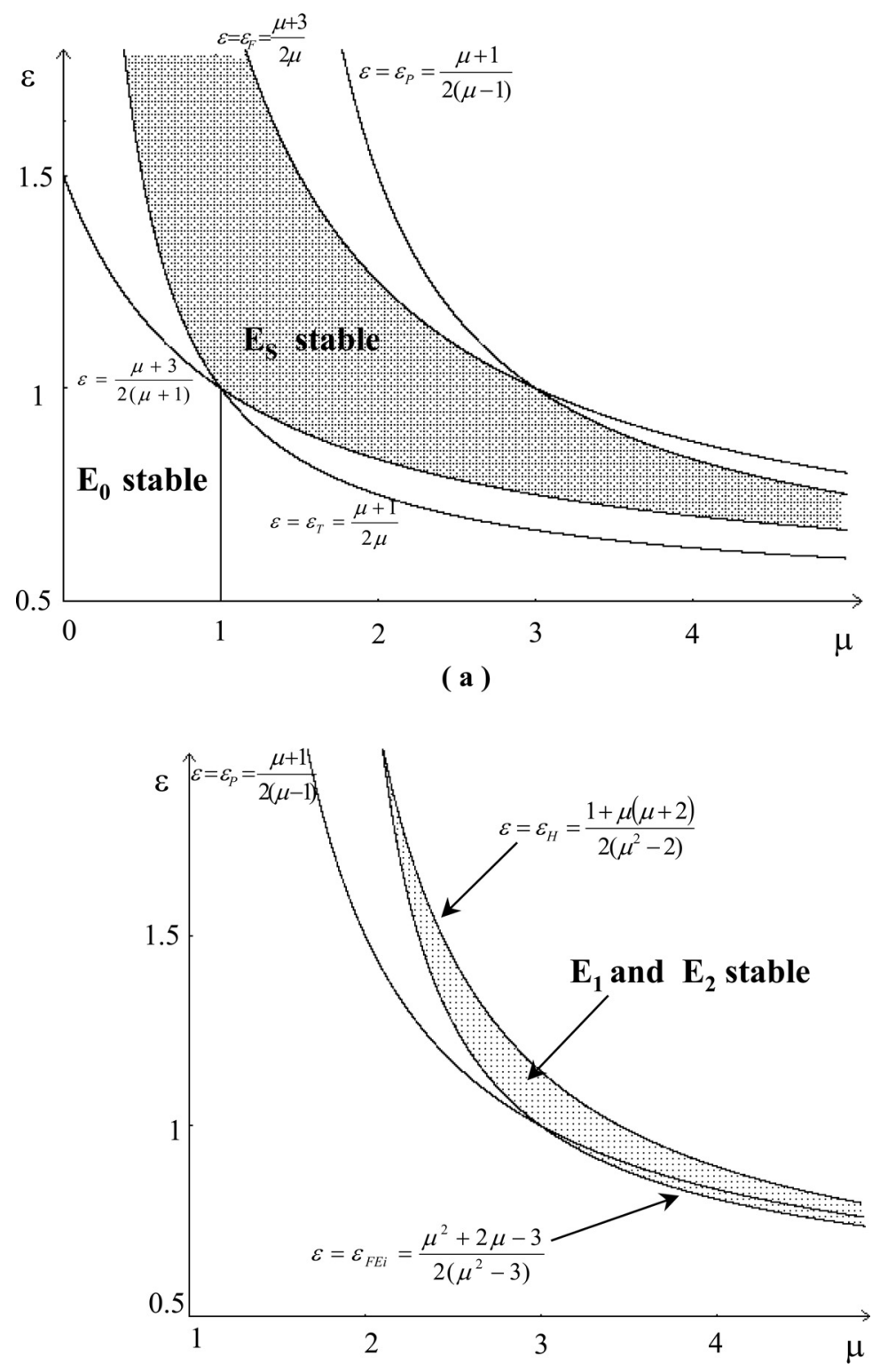

( b )

Fig. 2. Stability regions, in the parameter space $(\varepsilon, \mu)$, in the symmetric case of homogeneous players. (a) The stability region of the steady state $E_{S}$. (b) The stability region of the steady states $E_{1}$ and $E_{2}$.

i.e., every initial condition taken in the interior of the strategy space converges to it. So, the steady states $E_{1}$ and $E_{2}$ have been created as a consequence of demand overestimation. Moreover, it can be noticed that the steady state $E_{S}$, close to the Nash equilibrium $N E$, is unstable. 


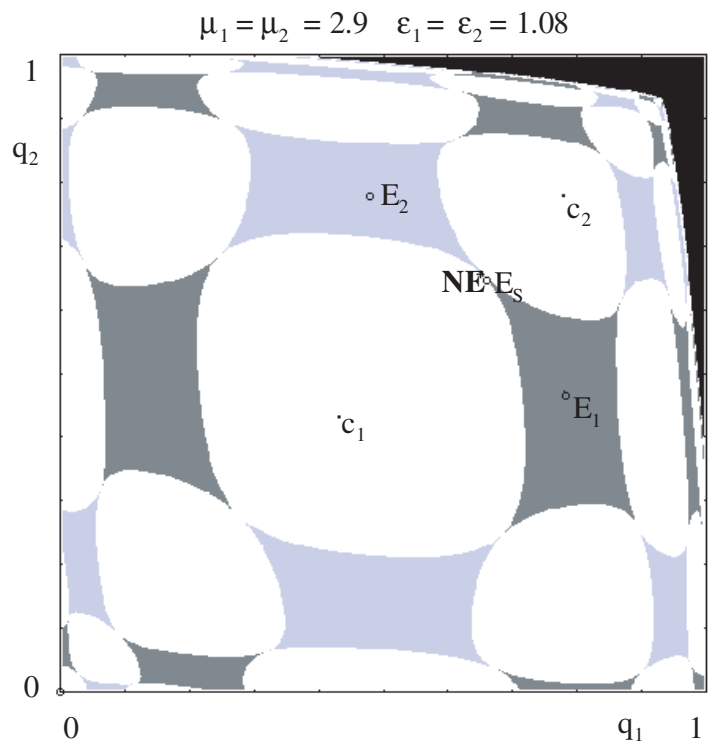

Fig. 3. Multiple steady states and their basins of attraction in the case of homogeneous players $E_{1}$ and $E_{2}$ are stable steady states, with basins represented by light and dark grey regions respectively. The initial conditions taken in the white regions generate paths that tend to a stable cycle of period 2 (the periodic points are denoted by $c_{1}$ and $c_{2}$ ), the initial conditions in the black region generate unfeasible trajectories. Profits and welfare in NE are $\Pi_{1}=\Pi_{2}=0.429$, $W=1.717$; Profits and welfare in $E_{1}$ are $\Pi_{1}=0.519, \Pi_{2}=0.247, W=1.549$; Profits and welfare in $E_{2}$ are $\Pi_{1}=0.247, \Pi_{2}=0.519, W=1.549$.

The profits of the two firms in the steady state $E_{1}=(0.78,0.472)$ are $\Pi_{1}=0.519$ and $\Pi_{2}=0.247$. Hence, firm 1 has a higher market share and earns a higher profit. The consumer surplus is $C S=0.784$ and welfare is $W=1.549$. In the symmetric Nash equilibrium $N E=(0.655,0.655)$ the profits are $\Pi_{1}=\Pi_{2}=0.429$, consumer surplus is $C S=0.858$ and welfare is, therefore, $W=1.717$. Obviously, welfare and industry output is higher in the full-information Nash equilibrium. However, note that the profit of firm 1 is higher than the profit it would earn in the Nash equilibrium and this firm, although unwittingly, has an advantage from not having perfect information about the true demand. The situation in $E_{2}$ is the same, although with the roles of firms 1 and 2 interchanged.

\subsection{Heterogeneous players: numerical investigation of global dynamics and bifurcations}

In this section the assumption of homogeneous players will be relaxed. We consider situations where the players differ with respect to their reaction functions $\left(\mu_{1} \neq \mu_{2}\right)$ and/or the scale of demand misspecification $\left(\varepsilon_{1} \neq \varepsilon_{2}\right)$. In the presence of such heterogeneities the symmetry properties of the map (29), which allowed us to obtain in Sec. 3.2 some analytical results on the existence, stability and local bifurcations 
of steady states, no longer hold. In the more general heterogeneous case even an analytic computation of the fixed points is not an easy task. However, it turns out that many of the results described in Sec. 3.2 continue to hold (mutatis mutandis) in the presence of heterogeneities, in particular, that situations of multistability arise as a consequence of variations of the "accuracy parameters" $\varepsilon_{i}$. So, in this subsection we employ a numerical approach that is guided by the previous results and some global geometric properties of the map (29). When several coexisting attractors are present, the boundaries that separate their basins of attraction will be studied numerically to gain some insights into their complex topological structure.

\subsubsection{Heterogeneity and differences in outcome}

As a starting point, let us consider a situation with homogeneous players, who know the true demand function: $\mu_{1}=\mu_{2}=2.9$ and $\varepsilon_{1}=\varepsilon_{2}=1$. The unique Nashequilibrium, $N E=(1-1 / \mu, 1-1 / \mu) \simeq(0.655,0.655)$, is globally asymptotically stable, i.e., any initial condition chosen in the strategy space $\mathcal{S}=\{[0,1] \times[0,1]\}$ generates a trajectory that converges to $N E .^{\mathrm{h}}$

We will first consider the dynamics if the symmetry is broken by a slight misspecification of the demand only by player 2 and compare it with the case of homogeneous players. We will continue to denote the fixed points of (29) as $E_{S}, E_{1}$ and $E_{2}$, although the reader should be aware that their actual coordinates now differ from those given in (36) and (38). Of course, as before the fixed points do not coincide with $N E$. For $\varepsilon_{2}=1.05$ we observe that $E_{S}$ loses its stability via a flip bifurcation (at which it becomes a saddle point), and a stable cycle of period 2 is the unique attractor. If the parameter $\varepsilon_{2}$ is further increased, a pitchfork bifurcation of $E_{S}$ occurs at $\varepsilon_{2} \simeq 1.052$, at which two saddle fixed points are created. At this stage the stable cycle of period 2 is still the unique attractor. However, if $\varepsilon_{2}$ is increased further, the steady state $E_{2}$ (which is characterized by a higher quantity for player 2) becomes stable via a subcritical flip bifurcation. For instance, if $\varepsilon_{2}=1.08$ (player 2 overestimates demand by $8 \%$ ), and $\varepsilon_{1}=1$ (player 1 knows the true demand function), then for a set of initial quantities player 2, although unwittingly, achieves market dominance. This situation is depicted in Fig. 4(a), where the light grey regions represent the basin of attraction of the steady state $E_{2}$, denoted $\mathcal{B}\left(E_{2}\right)$. The white region represents the basin of attraction of the stable cycle of period 2, denoted $\mathcal{B}\left(C_{2}\right)$, where the 2-cycle $C_{2} \simeq((0.502,0.542),(0.720,0.777))$ is represented by two black dots in the figure. The strategy space $\mathcal{S}$ is no longer trapping. There exists a small region inside $\mathcal{S}$, the black region in Fig. 4, whose points generate diverging and negative trajectories. As these trajectories leave the strategy space, we will say that these points belong to the basin of infinity, $\mathcal{B}(\infty)$. In the

${ }^{\mathrm{h}}$ Indeed, if $\mu_{i} \in[0,4], i=1,2$, and $\varepsilon_{i}=1, i=1,2$, then the region $\mathcal{S}$ is trapping, i.e., any trajectory starting inside $\mathcal{S}$ is entirely included inside $\mathcal{S}$, as $\tilde{T}\left(q_{1}, q_{2}\right)=\left(\mu_{1} q_{2}\left(1-q_{2}\right), \mu_{2} q_{1}\left(1-q_{1}\right)\right) \in$ $\left[\mu_{1} / 4, \mu_{2} / 4\right] \subseteq \mathcal{S}$ for each $\left(q_{1}, q_{2}\right) \in \mathcal{S}$. 

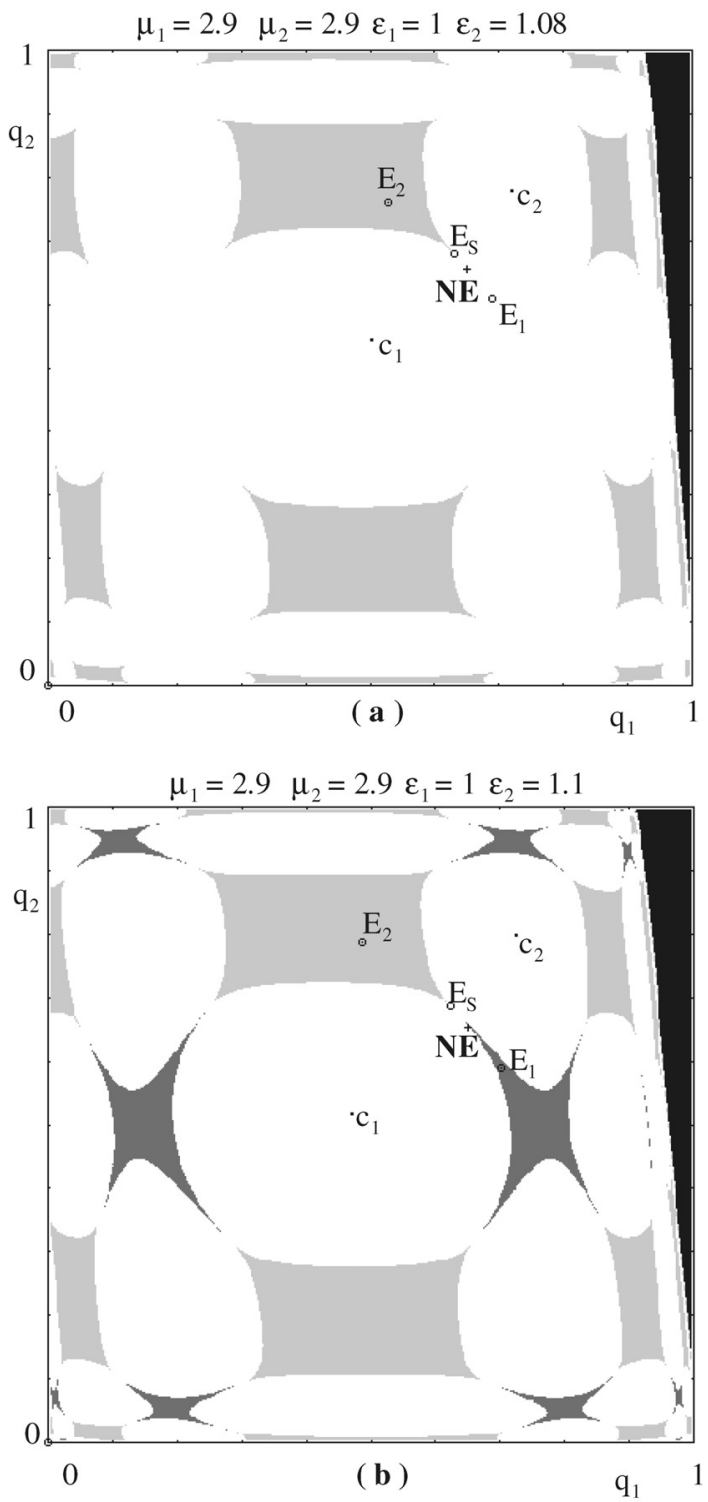

Fig. 4. Multiple steady states and their basins of attraction - heterogeneous players. Profits and welfare in NE: $\Pi_{1}=\Pi_{2}=0.429, W=1.717$. Panel (a): Profits and welfare in $E_{2}: \Pi_{1}=0.278$, $\Pi_{2}=0.521, W=1.629$. Panel (b): Profits and welfare in $E_{1}: \Pi_{1}=0.493, \Pi_{2}=0.368, W=1.694$. Profits and welfare in $E_{2}: \Pi_{1}=0.237, \Pi_{2}=0.521, W=1.568$.

figures $N E$ denotes the Nash equilibrium of the "true" game. A comparison with the case of homogeneous players (where both players overestimate the true demand by the same factor) surprisingly shows that the asymmetry in the accuracies of the knowledge of players actually hurts the player who knows the true demand function. 
The profits of the firms in $E_{2}$ are $\Pi_{1}=0.278$ and $\Pi_{2}=0.521$ respectively. Recalling that in the Nash equilibrium $N E$ the profits are $\Pi_{1}=\Pi_{2}=0.429$, obviously player 2 not only gets a higher market share but also earns a higher profit than player 1 . On the other hand, as in the homogeneous case overall welfare in $E_{2}$ is $W=1.629$ and hence lower than in the full information Nash equilibrium.

\subsubsection{Topological structure of basins of attraction}

The topological structure of the basins is also sensitive to the parameters $\varepsilon_{i}$ and $\mu_{i}$ and needs to be studied if the dynamics of the map are to be fully understood. In particular consider the structure of $\mathcal{B}\left(E_{2}\right)$ in Fig. 4(a), obtained with parameters $\mu_{1}=\mu_{2}=2.9, \varepsilon_{1}=1$ and $\varepsilon_{2}=1.08$, which consists of several nonconnected portions nested inside $\mathcal{B}(C)$, the basin of the two-cycle $C=\left(c_{1}, c_{2}\right)$. This kind of basin structure is specific to noninvertible maps, which are transformations mapping distinct points into the same point (see the Appendix B for more details, see also Mira et al., 1996a, Abraham et al., 1997). ${ }^{\mathrm{i}}$

If $\varepsilon_{2}$ is further increased, the basin structure becomes even more complex. The steady state $E_{1}$ also becomes stable (via the subcritical flip bifurcation), and the situation illustrated in Fig. 4(b) for $\varepsilon_{2}=1.1$ is obtained. The dark-grey region represents the basin $\mathcal{B}\left(E_{1}\right)$ of the equilibrium $E_{1}$, where firm 1 has a higher market share. Now, similar to the homogeneous case we observe three coexisting attractors: the two locally stable equilibria $E_{1}$ and $E_{2}$, each with its own non connected basin, and a stable cycle of period two. Such a situation of a quite complex topological structure of the basins is typical if multistability (i.e., coexistence of several attractors) occurs. Notice the difference in the three long run patterns caused by the demand misspecification of only one player. In $E_{2}$ player 2 has a higher market share and the profits are $\Pi_{1}=0.237$ and $\Pi_{2}=0.521$. The welfare in $E_{2}$ is $W=1.568$ and again smaller than in the Nash equilibrium. In $E_{1}$ player 1's market share is larger, and $\Pi_{1}=0.493$ and $\Pi_{2}=0.368$. Although welfare is again smaller than in the Nash equilibrium, $W=1.694$, the sum of the profits of the two players is larger than in NE. For a large set of initial quantities (the white region in Fig. 4(b)) the long run pattern is periodic and the two players behave in a quasi-synchronized way, as the periodic points are close to $\Delta$. It is also worth pointing out that the equilibrium

\footnotetext{
${ }^{i}$ The basic mechanism leading to the creation of non-connected basins, like $\mathcal{B}\left(E_{2}\right)$ in Fig. 3(a), is a consequence of the fact that the iterated map $\tilde{T}$ is a noninvertible map (see the Appendix $\mathrm{B}$ for definitions and properties concerning noninvertible maps). Let $A$ be an attractor for the iterated map $\tilde{T}$. This means that a neighborhood $U(A)$ exists whose points converge to $A$. Of course $U(A) \subseteq$ $\mathcal{B}(A)$, but also the points of the phase space which are mapped inside $U$ after a finite number of iterations belong to $\mathcal{B}(A)$, so that the total basin of $A$ is given by $\mathcal{B}(A)=\bigcup_{n=0}^{\infty} \tilde{T}^{-n}(U(A))$, where $\tilde{T}^{-n}(x)$ represent the set of the rank- $n$ preimages of $x$ (i.e., the points mapped into $x$ after $n$ repeated applications of $\tilde{T}$ ). If $\tilde{T}$ is a noninvertible map, then the total basin may be nonconnected because if $U(A)$ (or its preimages) belongs to regions whose points have several distinct preimages, the action of the distinct inverses, may give preimages of $U(A)$ which are disjoint from $U(A)$. Due to the unfolding process of the Riemann foliation (see the Appendix B) under the action of the several distinct inverses, those preimages might be far away from $U(A)$.
} 


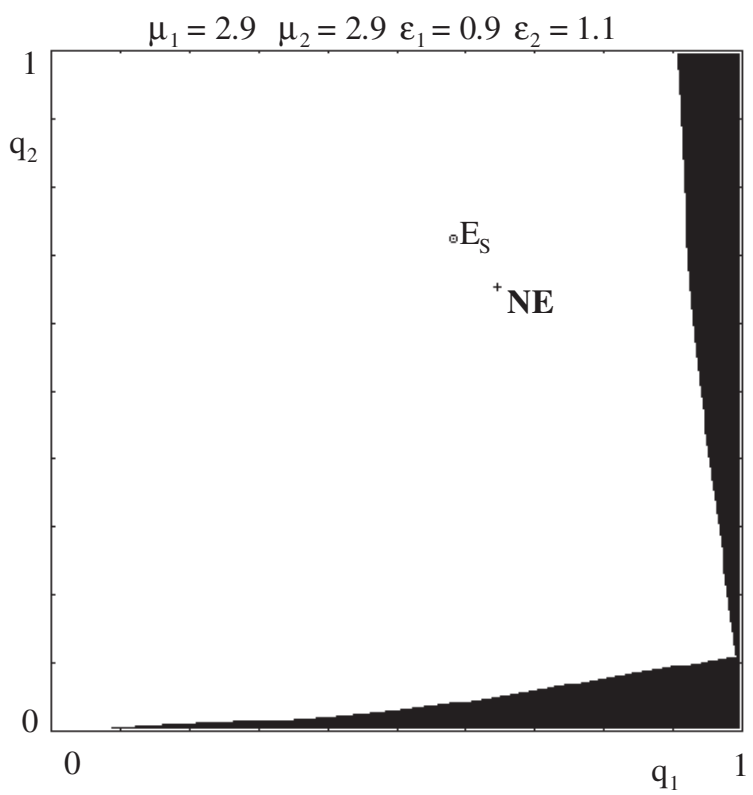

Fig. 5. The stabilizing effect of opposite misspecification of demand.

$E_{1}$ in Fig. 4(b), which just attains stability, is not robust in the following sense: it is very close to the boundary of its basin and not robust to exogenous perturbations. Even if the total basin $\mathcal{B}\left(E_{1}\right)$ has a considerable extension inside $\mathcal{S}$, the trajectories starting (or passing) at points located in a small neighborhood of $E_{1}$ may escape the basin e.g., as a consequence of a very small exogenous noise (thus causing an irreversible departure from $E_{1}$ ).

It is of interest to consider how the long run behavior is affected if one player overestimates the demand, whereas the other player underestimates it. As an example consider Fig. 5, where as before $\mu_{1}=\mu_{2}=2.9$, but $\varepsilon_{1}=0.9$ and $\varepsilon_{2}=1.1$. In this situation only one steady state, namely $E_{S}$, exists and all feasible (i.e., non diverging) trajectories converge to it. These observations seem to suggest that some stabilizing effect occurs if the players' misspecification of demand is in opposite directions (this insight is also confirmed by other numerical simulations). Furthermore, $E_{S}$ is close to the Nash equilibrium, the industry output is basically the same $(q=1.31)$ and welfare is almost as high $(W=1.691)$.

\subsection{Non-identical reaction functions}

Up to now, we have restricted our analysis to players with identical reaction functions $\left(\mu_{1}=\mu_{2}\right)$. We now briefly describe some numerical results obtained when the interfirm externality is asymmetric, which might be interpreted as a difference in 

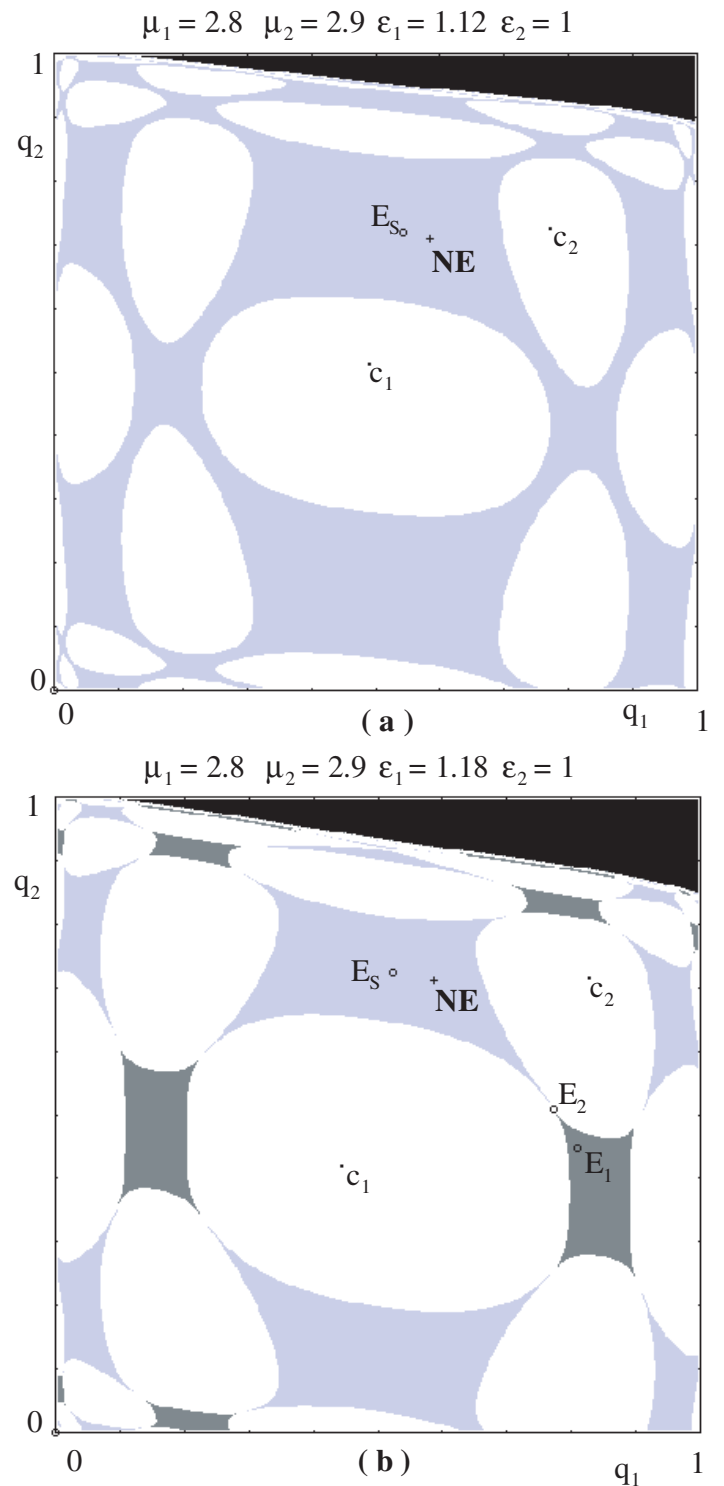

Fig. 6. The effect of non-identical reaction functions profits and welfare in $\mathrm{NE}: \Pi_{1}=0.338$, $\Pi_{2}=0.498, W=1.665$. Panel (a): Profits and welfare in $E_{S}: \Pi_{1}=0.318, \Pi_{2}=0.518, W=1.632$. Panel (b): Profits and welfare in $E_{1}: \Pi_{1}=0.465, \Pi_{2}=0.2, W=1.455$. Profits and welfare in $E_{S}: \Pi_{1}=0.312, \Pi_{2}=0.524, W=1.612$.

firms' abilities to gain an advantage from actions from the other firm. Firms' reaction functions have now slightly different heights as the values of the parameters $\mu_{i}$ differ slightly. As in the previous sequence of numerical simulations, we start from the benchmark case (26), i.e., $\varepsilon_{1}=\varepsilon_{2}=1$, with a set of parameters at which $N E$ is 
the unique, globally stable (Nash) equilibrium. This is obtained, for example, with $\mu_{1}=2.8, \mu_{2}=2.9$. If we now increase $\varepsilon_{1}$, first a stable cycle of period 2 appears due to a saddle-node bifurcation, and dynamic situations similar to the one shown in Fig. 6(a), obtained with $\varepsilon_{1}=1.12$ and $\varepsilon_{2}=1$, are observed. In this case the strategy space is constituted by the basins of the two coexisting attractors: the equilibrium $E_{S}$ and a two-cycle $C=\left(c_{1}, c_{2}\right.$ ) (as well as a small portion of $\mathcal{B}(\infty)$ ). Also in this case, as $\varepsilon_{1}$ is further increased, two new steady states are created, say again $E_{1}$ and $E_{2}$. The difference with respect to the situations considered before is that these new equilibria are created via a saddle-node bifurcation (through a mechanism similar to the one shown in Fig. 1). As a result these new steady states appear far away from $E_{S}$, and one is stable (a stable node) and one is unstable. Since these new steady states are located quite far away from the Nash equilibrium, a welfare comparison is even more important. For example, the situation for $\varepsilon_{1}=1.18$ is represented in Fig. 6(b). Two stable steady states coexist, $E_{S}$ and $E_{1}$, where the latter is a stable node. Furthermore, a stable cycle $C_{2}$ coexists. ${ }^{j}$ However, despite this difference in the kind of bifurcation which creates the fixed points, the global dynamic scenario obtained is quite similar to the one shown in the case of equal reaction functions, see e.g., Fig. 4(b). The Nash equilibrium $N E$ is located in the basin and near to $E_{S}$, industry output and welfare in the perceived equilibrium $E_{S}$ is again smaller. ${ }^{\mathrm{k}}$ If play converges to $E_{1}$, then firm 1 has a higher market share and profit $\left(\Pi_{1}=0.465\right)$ than firm $2\left(\Pi_{2}=0.2\right)$. Welfare, however, is considerably smaller in $E_{1}$, namely $W=1.455$, which is mainly caused by the very low profit of firm 2 in this situation. Again note that it is player 1 who overestimates demand and, despite the fact that player 2 knows the true demand, achieves (although unwittingly) not only market dominance, but gains a higher profit than in the full information case, whereas firm 2's profit is reduced by more than $50 \%$.

\section{A Duopoly Model with Misspecified Demand and Inertia}

In the previous section we assumed that players, although they use misspecified demand functions, always produce the quantities which maximize their expected profits. One might argue, however, that in such a situation of uncertainty players might exhibit some lack of confidence in their computed "best replies" and show some bias towards the status quo. In other words, their uncertainty about the actual customer behavior leads the players to adjust their quantities only partially to the best response. Recent experimental evidence by Rassenti et al. (2000) and Huck et al. (1999) suggests that such partial adjustment to the best response describes the behavior of real decision makers quite well. A different interpretation

\footnotetext{
jThe other fixed point $E_{2}$ is an unstable node located on the boundary among the three basins $\mathcal{B}\left(E_{1}\right), \mathcal{B}\left(E_{S}\right)$ and $\mathcal{B}\left(C_{2}\right)$.

k The profits of the firm in the Nash equilibrium are $\Pi_{1}=0.338$ and $\Pi_{2}=0.498$ respectively. The welfare is $W=1.665$. Profits and welfare in $E_{S}$ are similar, $\Pi_{1}=0.312, \Pi_{2}=0.524$ and $W=1.612$.
} 
of inertia of the agents in our model is that the players are exhibiting a lower degree of rationality than the agents using best reply dynamics.

Recalling that the best reply behavior is represented by (6), if we now assume that players only partially adjust their quantities towards the (perceived) best replies, we have to replace these equations with (see also Kopel, 1996, Puu, 1991, Huang, 2001)

$$
\begin{aligned}
& q_{1}(t)=q_{1}(t-1)+\lambda_{1}\left(\tilde{R}_{1}\left(q_{2}^{e, p r i o r}(t)\right)-q_{1}(t-1)\right), \\
& q_{2}(t)=q_{2}(t-1)+\lambda_{2}\left(\tilde{R}_{2}\left(q_{1}^{e, p r i o r}(t)\right)-q_{2}(t-1)\right),
\end{aligned}
$$

or equivalently with

$$
\begin{aligned}
& q_{1}(t)=\left(1-\lambda_{1}\right) q_{1}(t-1)+\lambda_{1} \tilde{R}_{1}\left(q_{2}^{e, p r i o r}(t)\right) \\
& q_{2}(t)=\left(1-\lambda_{2}\right) q_{2}(t-1)+\lambda_{2} \tilde{R}_{2}\left(q_{1}^{e, p r i o r}(t)\right) .
\end{aligned}
$$

The parameters $\lambda_{i} \in[0,1], i=1,2$, represent the speeds of adjustment and can be seen as a measure of the inertia: players exhibit no inertia for $\lambda_{i}=1$ and increasing inertia for decreasing values of $\lambda_{i}$. The other Eqs. (15)-(17), of course, remain the same, since they describe the price formation process and the expectation updating, respectively. Analogously to (18) and (19), the resulting dynamical system can be reduced to

$$
\begin{aligned}
& q_{1}(t+1)=\left(1-\lambda_{1}\right) q_{1}(t)+\lambda_{1} \tilde{R}_{1}\left(\tilde{D}_{1}\left(f\left(q_{1}(t)+q_{2}(t)\right)\right)-q_{1}(t)\right), \\
& q_{2}(t+1)=\left(1-\lambda_{2}\right) q_{2}(t)+\lambda_{2} \tilde{R}_{2}\left(\tilde{D}_{2}\left(f\left(q_{1}(t)+q_{2}(t)\right)\right)-q_{2}(t)\right) .
\end{aligned}
$$

It is important to notice that the introduction of inertia does not change the fixed points: setting $q_{i}(t+1)=q_{i}(t)$ in Eqs. (43) and (44) gives the same conditions as in the previous section. Consequently, the profits and welfare properties of the long run outcomes are unchanged by the inertia of players.

If we again consider the special case (20), i.e., player 2 knows the true demand function and player 1's misspecified demand function is $\tilde{D}_{1}(p)=\varepsilon D(p)$, we have

$$
q_{2}(t+1)=\left(1-\lambda_{2}\right) q_{2}(t)+\lambda_{2} R_{2}\left(q_{1}(t)\right)
$$

Furthermore, using the same arguments as before, we get

$$
q_{1}(t+1)=\left(1-\lambda_{1}\right) q_{1}(t)+\lambda_{1} \varepsilon R_{1}\left[\frac{\varepsilon-1}{\varepsilon} q_{1}(t)+q_{2}(t)\right] .
$$

Of course, if both players have misspecified demand functions, then a similar equation is derived for player 2 . 


\subsection{Global dynamics and the influence of inertia}

If we again, as in Sec. 3, use a linear inverse demand curve and cost functions with interfirm externalities, the best replies are quadratic. Hence, the dynamics in this case are governed by the map

$$
\tilde{T}_{\lambda}:\left\{\begin{aligned}
q_{1}(t+1)= & \left(1-\lambda_{1}\right) q_{1}(t)+\lambda_{1} \mu_{1}\left[\varepsilon_{1} q_{2}(t)\left(1-q_{2}(t)\right)\right. \\
& \left.+\left(\varepsilon_{1}-1\right) q_{1}(t)\left(1-\frac{\varepsilon_{1}-1}{\varepsilon_{1}} q_{1}(t)-2 q_{2}(t)\right)\right] \\
q_{2}(t+1)= & \left(1-\lambda_{2}\right) q_{2}(t)+\lambda_{2} \mu_{2}\left[\varepsilon_{2} q_{1}(t)\left(1-q_{1}(t)\right)\right. \\
& \left.+\left(\varepsilon_{2}-1\right) q_{2}(t)\left(1-\frac{\varepsilon_{2}-1}{\varepsilon_{2}} q_{2}(t)-2 q_{1}(t)\right)\right] .
\end{aligned}\right.
$$

Of course, for $\lambda_{i}=1, i=1,2$ this map reduces to $\tilde{T}$ given in Eq. (29). In the following analysis we investigate how the dynamics of (45) depend on the parameters $\lambda_{i}$.

Again, in the symmetric case, i.e., $\mu_{1}=\mu_{2}=\mu, \varepsilon_{1}=\varepsilon_{2}=\varepsilon$ and $\lambda_{1}=\lambda_{2}=\lambda$, we obtain a map which is symmetric with respect to the diagonal $\Delta$. The dynamics embedded into the invariant submanifold $\Delta$ is governed by the one-dimensional map

$$
q(t+1)=[1-\lambda+\lambda \mu(2 \varepsilon-1)] q-\frac{\lambda \mu}{\varepsilon}(2 \varepsilon-1)^{2} q^{2},
$$

which is conjugate to the standard logistic map $z=a z(1-z)$, with

$$
a=1-\lambda+\lambda \mu(2 \varepsilon-1)
$$

by the linear transformation

$$
q=\frac{\varepsilon(1-\lambda+\lambda \mu(2 \varepsilon-1))}{\lambda \mu(2 \varepsilon-1)^{2}} z .
$$

The fixed points of the map (45) do not depend on the parameters $\lambda_{i}$ and, hence, are the same as those of the map $\tilde{T}$ given by the solutions of the algebraic system (31). A study of the Jacobian matrix of $\tilde{T}_{\lambda}$ (see the appendix) reveals that the analysis of the local stability and the local bifurcations of the steady states in the case of homogeneous players is similar to the one described in Sec. 3.2. In particular, the condition for the pitchfork bifurcation of $E_{S}$ in the direction perpendicular to $\Delta$, at which the two equilibria $E_{1}$ and $E_{2}$ are created, is exactly the same, i.e., $\varepsilon=\varepsilon_{P}$. On the other hand, the condition for the flip bifurcation of $E_{S}$ along $\Delta$, at which a stable cycle of period 2 is created, is influenced by the parameter $\lambda$, and occurs at

$$
\varepsilon=\varepsilon_{F, \lambda} \equiv \frac{2+\lambda(\mu+1)}{2 \lambda \mu} .
$$

This can also be easily deduced from the restriction $\left.\tilde{T}_{\lambda}\right|_{\Delta}$ given in Eq. (46), whose first flip bifurcation occurs for $a=3$, with $a$ given in Eq. (47). The difference between the two values of $\varepsilon$ in Eqs. (48) and (41) is given by $\varepsilon_{F, \lambda}-\varepsilon_{F}=(1-\lambda) / \lambda \mu$. 
From this expression it is easy to see that for a given value of $\mu$ the creation of stable cycles along $\Delta$ occurs for increasing values of the "misspecification" parameter $\varepsilon$ if $\lambda$ is decreasing in $(0,1)$. This can be interpreted by saying that in the homogeneous case increasing inertia has some kind of dampening effect since it inhibits the occurrence of endogenous oscillations (periodic or chaotic) which would occur for higher values of $\varepsilon$. This feature is also observed in the numerical explorations performed in the case of heterogeneous players. However, these numerical results also reveal that the other route to complexity, which is related to the complex structure of the basins of attraction of coexisting stable steady states, may be still observed.

In order to see this, we take the sets of parameters $\mu_{i}$ and $\varepsilon_{i}$ used in the previous section and focus on the role of the inertia parameters $\lambda_{i}$. This enables us to directly compare the dynamics of the two cases. We start our numerical explorations with $\mu_{1}=\mu_{2}=\mu=2.9, \varepsilon_{1}=1, \varepsilon_{2}=1.08$ and $\lambda_{1}=0.7, \lambda_{2}=0.6$, which means that player 1 has a lower degree of inertia than player 2 and knows the demand curve, whereas player 2 overestimates the demand by $8 \%$. Figure 7 (a) depicts this situation. There are three positive steady states. The steady state $E_{1}$ with basin $\mathcal{B}\left(E_{1}\right)$ (which is represented by the dark-grey region), the steady state $E_{2}$ with basin $\mathcal{B}\left(E_{2}\right)$ (represented by the light-grey region), and $E_{S}$, which is a saddle point. The stable set of $E_{S}$ constitutes the boundary which separates the two basins. This contrasts with the model without inertia (compare with Fig. 4(a)) as no stable cycles exist, but the perceived equilibrium $E_{1}$ is stable. The only kind of long-run dynamics is given by one of the equilibria $E_{1}$ and $E_{2}$, where $E_{1}$ has a very large basin. The fact that players show inertial behavior now benefits player 1 , and for most initial choices player 1 , who has perfect knowledge of the demand function, benefits in the long run. In the equilibrium $E_{1}$ player 1 has a higher market share and earns a higher profit than player $2\left(\Pi_{1}=0.477, \Pi_{2}=0.384\right)$, whereas in the case without inertia only the equilibrium $E_{2}$ is stable, where firm 2 dominates the market. If the degree of inertia of player 2 is decreased, the basin $\mathcal{B}\left(E_{2}\right)$ enlarges. So, obviously, the difference between the degrees of inertia between the players matter for the long run outcome of the game.

As a final observation we want to point out that the basin $\mathcal{B}\left(E_{2}\right)$ may even undergo some global (or contact) bifurcations which cause qualitative changes in its topological structure. For example, by increasing $\lambda_{2}$ from 0.6 to 0.65 (see Fig. $7(\mathrm{~b})$ ) we observe an important change in the structure of $\mathcal{B}\left(E_{2}\right)$ : it is transformed from a simply connected set into a non connected set, where the latter is formed by several disjoint portions nested inside $\mathcal{B}\left(E_{1}\right)$. This is a typical contact bifurcation described in textbooks on noninvertible maps (see e.g., Mira et al., 1996a, Abraham et al., 1997). Here we just give a general qualitative description of the mechanism that leads at the formation of non-connected portions of a basin of attraction, see Appendix B for more details. Such bifurcation is due to a contact between the boundary which separates the basins, that is, the stable set $W^{s}\left(E_{S}\right)$ of the saddle

\footnotetext{
${ }^{1}$ Note also that the strategy space is trapping.
} 

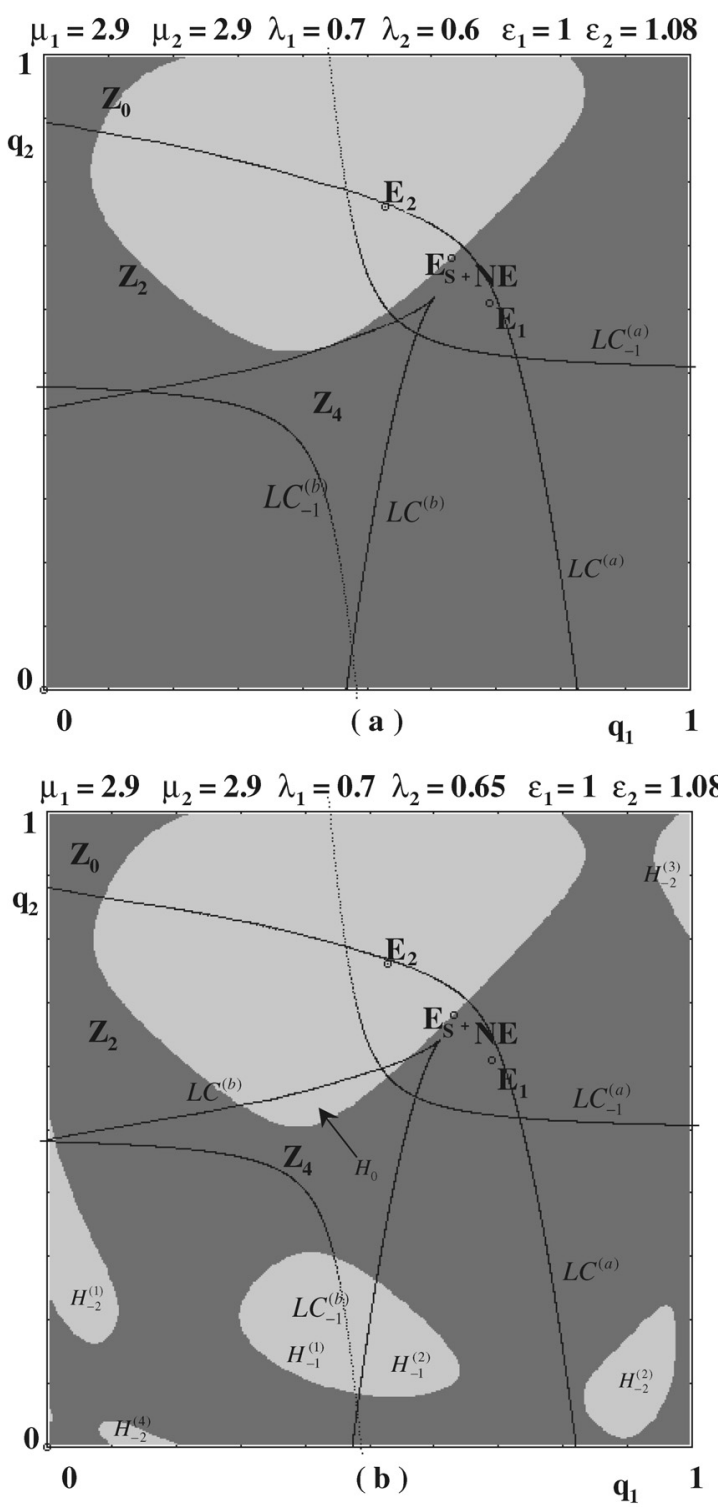

Fig. 7. The effect of inertia Profits and welfare in NE: $\Pi_{1}=\Pi_{2}=0.429, W=1.717$; Profits and welfare in $E_{1}: \Pi_{1}=0.477, \Pi_{2}=0.384, W=1.705$.

point $E_{S}$ and the critical curve $L C^{(b)}$ which separates two regions, denoted by $Z_{2}$ and $Z_{4}$ in Fig. 7 , whose points have two and four preimages respectively.

In fact, a map $T$ is noninvertible if there exist distinct points that have the same image. This means that several rank-1 preimages of a given point may exist and, accordingly, the space can be subdivided into regions $Z_{k}, k \geq 0$, whose points have $k$ distinct rank-1 preimages. These regions are separated by critical curves 

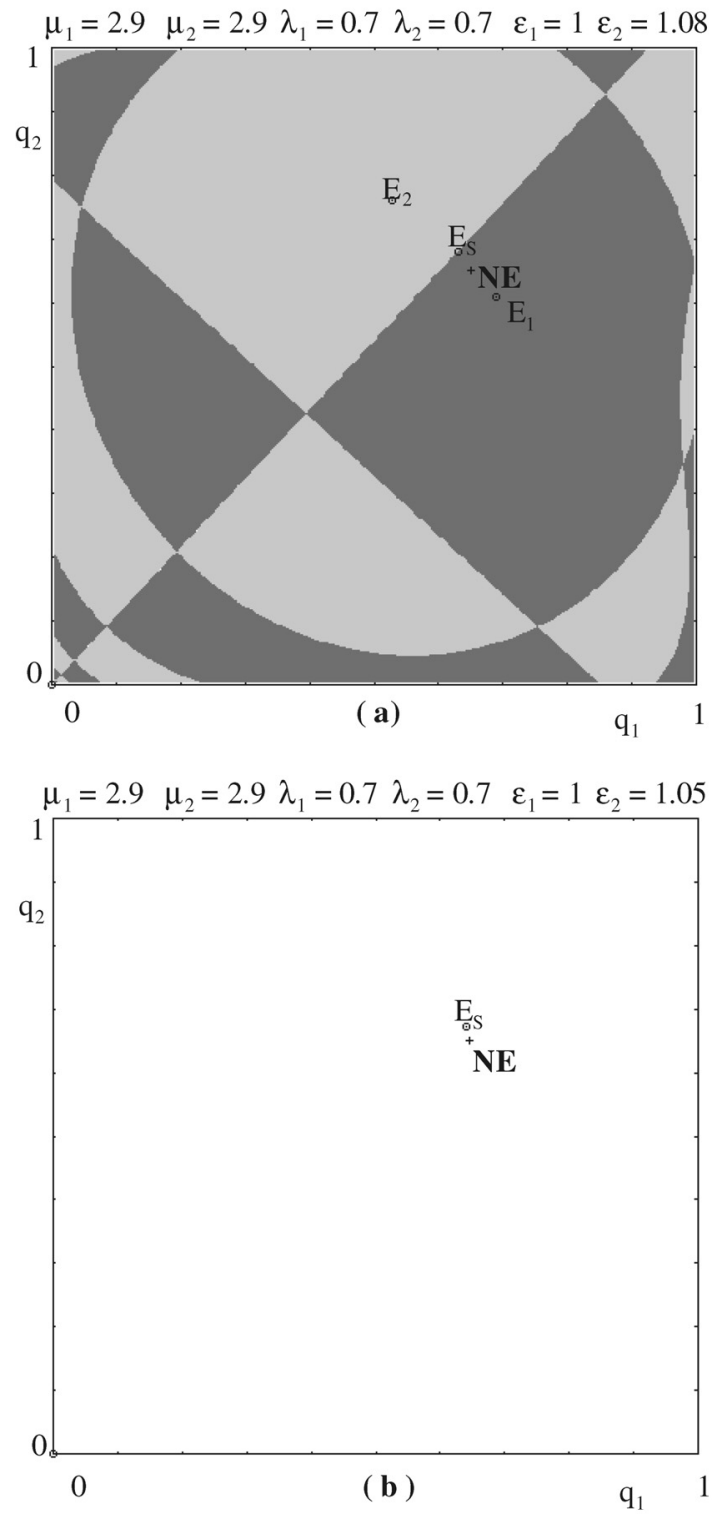

Fig. 8. Players have the same degree of inertia. Firm 1 has a correct knowledge of the demand function, whereas firm 2 overestimated the demand. (a) Two stable subjective equilibria E1 and E2 exist. (b) By decreasing the demand overestimation of firm 2, the unique attractor is the subjective equilibrium ES, close to the Nash equilibrium NE.

(see the Appendix B). This helps us to understand the basic mechanism for the creation of non-connected basins. We recall that the basin of an attractor $A$ is the set of all the points that generate trajectories converging to $A$. So, if $U$ is a neighborhood of $A$ whose points converge to $A$ (which exists by definition of an 
attractor) then $U \subseteq \mathcal{B}(A)$, and also the points which are mapped inside $U$ after a finite number of iterations belong to $\mathcal{B}(A)$, thus the basin of $A$ is formed by all the preimages of the points of $U$, i.e., $\mathcal{B}(A)=\bigcup_{j=0}^{\infty} T^{-j}(U)$, where $T^{-1}(x)$ represents the set of the rank-1 preimages of $x$ (i.e., the points mapped into $x$ by $T$ ), and $T^{-j}(x)$ represents the set of the rank-j preimages of $x$ (i.e., the points mapped into $x$ after $j$ applications of $T$ ). If we assume that $\mathcal{B}(A)$ is a connected basin for a given set of parameters, and as a parameter is changed $\mathcal{B}(A)$ has a contact with a critical curve after which a portion of $\mathcal{B}(A)$ enters a region with more preimages, then new portions of $\mathcal{B}(A)$ will be created after the contact, thus giving a sudden enlargement of the basin due to the creation of new non-connected portions (also called islands in Mira et al., 1996).

Finally, let us consider what happens if the degrees of inertia of the players are equal, i.e., $\lambda_{1}=\lambda_{2}$. In this case the two basins approximately occupy an equal portion of the strategy space (see Fig. 8(a), where $\lambda_{1}=\lambda_{2}=0.7$ ). Moreover, the two basins have a quite complicated structure. Much simpler dynamics are obtained if the overestimation of demand by player 2 is less strong. For example, in Fig. 8(b), where $\varepsilon_{2}=1.05$, only one steady state exists (close to the Nash equilibrium $N E$ ), which is globally asymptotically stable in the strategy space $\mathcal{S}$. Recall that for the same sets of parameters $\mu_{i}$ and $\varepsilon_{i}, i=1,2$, in the case without inertia, i.e., $\lambda_{i}=1$ $(i=1,2)$, a stable cycle of period 2 as unique attractor occurs, as described in Sec. 3.3.

\section{Conclusion}

In this paper we have analyzed a quantity-setting duopoly game where firms do not know the true demand relationship, but use some conjecture of it instead. Two versions of firm behavior in this duopoly game have been considered here. In the first version, players choose their quantities such that their (perceived) profits are maximized. In the second version it is assumed that players exhibit some kind of bias towards their current production choice and only partially adjust to the (perceived) best reply. Both scenarios have been compared to the situation where players know the demand function. For the sake of illustration, we used a linear inverse demand curve and quadratic reactions functions that can be obtained by choosing nonlinear cost functions including an interfirm externality. This choice enabled us to give a variety of results based on a combination of analytical and numerical arguments.

It turns out that although in the game which represents the true situation a unique, globally stable Nash equilibrium exists, if players use a misspecified demand function and/or exhibit inertia, the long run outcome of the game may be quite different. Multiple equilibria may occur in the perceived game and coexist with other long run outcomes (cycles and even chaotic attractors). The performance properties (profits and welfare) in these equilibria may be quite different than in the Nash equilibrium. Furthermore, these equilibria may have quite complex basins of attraction. We have also briefly described the mechanism which is responsible for the 
creation of the complicated structure of the basins of attraction, by referring to the role of noninvertibility of the two-dimensional map generating the dynamics of the game. For economists, the significance of observing such a complicated structure of the basins of the equilibria stems from the fact that the outcome of the game strongly depends on the initial conditions. The same game starting from nearby initial conditions may end up at different perceived equilibria with quite different welfare properties and often far away from the Nash equilibrium of the true game.

It is important to stress that when the economy ends up in one of the equilibria, the agents have no reason to seek to change their behavior, because the economic outcome they observe is perfectly consistent with their beliefs. In fact, they have no way of becoming aware of the overall welfare loss that is occurring, and indeed usually one player undergoes a welfare gain compared to the full information Nash equilibrium. The welfare loss/gain results from the fact that each player is putting onto the market a quantity different from what they would offer in the full information case. This in turn leads to a different price than would prevail in the full information case. The welfare loss/gain is related to the value (at the market clearing price) of the quantity differences just referred to. It seems more likely that agents might seek to change behavior when the game converges to the cyclical outcomes that can sometimes occur (see e.g., Fig. 4). These outcomes should perhaps be seen as taking the model beyond its usable range, since here agents would surely seek to actively learn about the demand function. This is an obvious and important extension of the model developed here, but one that takes us beyond the aims and the scope of this paper, so it is left for future research. Attempts to incorporate learning into models with misspecified demand include Schinkel et al. (2002), Szidarovszky and Krawczyk (2003) and Wenzelburger (2003).

Finally we make the point that although we have provided a specific example with a linear demand function and quadratic reaction function, we may conjecture that the phenomena observed here would occur more generally in models with downward sloping demand functions and unimodal reaction functions as those are the ingredients that give rise to the multiple steady states and the noninvertible map.

\section{Acknowledgments}

We thank F.X. Hof for helpful comments and suggestions. We also acknowledge comments from participants at the CEF2002 meeting at Aix-en-Provence, the Conference on Complexity in Space and Time 2002, University of Southern Denmark, the MDEF 2002 Conference, University of Urbino and seminar participants in the Economics Department at Chuo University. The usual caveat applies. This work has been performed under the auspices of the national research project "Nonlinear models in economics and finance: complex dynamics, disequilibrium, strategic interactions", MIUR, Italy, and under the Joint Research Grant (0382): "Reconsideration of economic dynamics from a new perspective of nonlinear theory", Chuo University, Japan. This support is gratefully acknowledged. 


\section{Appendix A. Local Stability Analysis in the Case of Homogeneous Players}

In this appendix we describe the eigenvalues analysis that leads us to the characterization, shown in Fig. 2, of the stability domains, in the parameter space $\mu-\epsilon$, of the steady states $E_{S}, E_{1}$ and $E_{2}$, given in (36) and (37), of the map $\tilde{T}_{s}$ defined in (33). This analysis is based on the study of the eigenvalues of the Jacobian matrix of the two-dimensional map $\tilde{T}_{s}$, given by

$$
D \tilde{T}_{s}\left(q_{1}, q_{2}\right)=\left[\begin{array}{cc}
\mu(\varepsilon-1)\left(1-2 \frac{\varepsilon-1}{\varepsilon} q_{1}-2 q_{2}\right) & \mu\left(\varepsilon\left(1-2 q_{2}\right)-2(\varepsilon-1) q_{1}\right) \\
\mu\left(\varepsilon\left(1-2 q_{1}\right)-2(\varepsilon-1) q_{2}\right) & \mu(\varepsilon-1)\left(1-2 \frac{\varepsilon-1}{\varepsilon} q_{2}-2 q_{1}\right)
\end{array}\right],
$$

computed at the fixed points.

Let us note, first, that at any point $(q, q) \in \Delta$, the Jacobian $D \tilde{T}_{s}$ has the structure

$$
D T(q, q)=\left[\begin{array}{ll}
A & B \\
B & A
\end{array}\right]
$$

with $A=\mu(\varepsilon-1)\left(1-2 \frac{2 \varepsilon-1}{\varepsilon} q\right)$ and $B=\mu(\varepsilon-2(2 \varepsilon-1) q)$. Such a matrix has real eigenvalues, given by

$$
\begin{array}{ll}
z_{\|}(q)=A+B & \text { with eigenvector } \mathbf{r}_{\|}=(1,1) \text { along } \Delta, \\
z_{\perp}(q)=A-B & \text { with eigenvector } \mathbf{r}_{\perp}=(1,-1) \text { perpendicular to } \Delta .
\end{array}
$$

It is easy to see that the product of such matrices has the same structure, hence all the fixed points and the cycles embedded in the invariant diagonal $\Delta$ have real eigenvalues with eigenvectors along $\Delta$ and perpendicular to $\Delta$, respectively. This allows us to carry out a complete analysis of the local stability and bifurcations of the two symmetric steady states. In fact, from $z_{\|}(0)=\mu(2 \varepsilon-1)$ and $z_{\perp}(0)=-\mu$ we deduce that $E_{0}$ is stable for $\mu<1$ and $\varepsilon<(1+\mu) / 2 \mu$. So, if $E_{S}$ is positive then $E_{0}$ is unstable. Indeed, at $\varepsilon=\varepsilon_{T} \equiv(1+\mu) / 2 \mu$ we have $E_{0} \equiv E_{S}$ and if $\varepsilon$ is increased across $\varepsilon_{T}$ a transcritical bifurcation occurs along $\Delta$.

For the positive symmetric fixed point $E_{S}$ we have $z_{\|}\left(q_{S}\right)=2-\mu(2 \varepsilon-1)$ and $z_{\perp}\left(q_{S}\right)=\mu-2 /(2 \varepsilon-1)$. So, from the sufficient conditions for stability, $-1<z_{\|}\left(q_{S}\right)<1$ and $-1<z_{\perp}\left(q_{S}\right)<1$, we deduce that:

- $0<\mu \leq 1$ : $E_{S}$ is stable for $\varepsilon_{T}<\varepsilon<\varepsilon_{F}$, and at $\varepsilon_{F}$ a flip (or period doubling) bifurcation occurs at which $E_{S}$ becomes a saddle point, with unstable set along $\Delta$, and a stable cycle of period 2 is created along $\Delta .^{\mathrm{m}}$

- $1<\mu \leq 3: E_{S}$ is stable for $(\mu+3) /(2(\mu+1))<\varepsilon<\varepsilon_{F}$. For $\varepsilon_{T}<\varepsilon<$ $(\mu+3) /(2(\mu+1))$ the steady state $E_{S}$ is a saddle point, with stable set along $\Delta$ and unstable set perpendicular to $\Delta$. If $\varepsilon$ is increased beyond $\varepsilon_{P}$, a pitchfork

${ }^{m}$ Note that after the occurrence of the flip bifurcation transverse stability in the direction orthogonal to $\Delta$ is not changed. 
bifurcation in the direction $\mathbf{r}_{\perp}$ occurs at $\varepsilon_{P}>\varepsilon_{F}$, and $E_{S}$ becomes a repelling node (i.e., it becomes unstable also in the direction $\mathbf{r}_{\perp}$ ). Two new fixed points $E_{1}$ and $E_{2}$ are created, which are saddle points with stable set along $\mathbf{r}_{\perp}$ and unstable set along $\Delta$.

- If $\mu=3$ then $\varepsilon_{F}=\varepsilon_{P}=1$, i.e., if $\varepsilon$ is increased across the value 1 (the case of correct demand estimate) then the two bifurcations occur simultaneously, i.e., $E_{S}$ becomes a repelling node, two fixed points are created in the direction orthogonal to $\Delta$ and, simultaneously, a cycle of period 2 is created along $\Delta$.

- If $\mu>3$ we have $\varepsilon_{P}<\varepsilon_{F}$, so $E_{S}$ is stable for $(\mu+3) /(2(\mu+1))<\varepsilon<\varepsilon_{P}$. In this case, at $\varepsilon=\varepsilon_{P}$ a pitchfork bifurcation occurs at which the stable node $E_{S}$ becomes a saddle point with unstable set in the direction $\mathbf{r}_{\perp}$ and two stable fixed points, $E_{1}$ and $E_{2}$ are created. If $\varepsilon$ is increased beyond $\varepsilon_{P}$ a flip bifurcation along $\Delta$ occurs at $\varepsilon_{F}>\varepsilon_{P}, E_{S}$ becomes a repelling node, and a saddle-cycle of period 2 is created along $\Delta$.

In order to obtain the boundaries of the stability domain of the steady states $E_{1}$ and $E_{2}$, let us consider the Jacobian matrix (A.1) computed at $E_{1}$ and $E_{2}$, given by

$$
D \tilde{T}_{s}\left(E_{1}\right)=\left[\begin{array}{ll}
A_{1} & B_{1} \\
B_{2} & A_{2}
\end{array}\right] \text { and } D \tilde{T}_{s}\left(E_{2}\right)=\left[\begin{array}{ll}
A_{2} & B_{2} \\
B_{1} & A_{1}
\end{array}\right]
$$

respectively, where

$$
\begin{aligned}
& A_{1}=\frac{(\varepsilon-1)(1+\sqrt{\Psi(\varepsilon, \mu)}-2 \varepsilon)}{2 \varepsilon-1}, \quad A_{2}=\frac{(\varepsilon-1)(1-\sqrt{\Psi(\varepsilon, \mu)}-2 \varepsilon)}{2 \varepsilon-1}, \\
& B_{1}=\frac{\varepsilon(1+\sqrt{\Psi(\varepsilon, \mu)}-2 \varepsilon)}{2 \varepsilon-1}, \quad B_{2}=\frac{\varepsilon(1-\sqrt{\Psi(\varepsilon, \mu)}-2 \varepsilon)}{2 \varepsilon-1} .
\end{aligned}
$$

It is easy to see that $E_{1}$ and $E_{2}$ have the same characteristic equation, because the two matrices $D \tilde{T}_{s}\left(E_{i}\right), i=1,2$, have the same trace $\operatorname{Tr}=A_{1}+A_{2}=2(1-\varepsilon)$ and determinant det $=A_{1} A_{2}-B_{1} B_{2}=2 \varepsilon\left(\mu^{2}-2\right)-\mu(\mu+2)$. So, due to the symmetry of the map $\tilde{T}_{s}$, the two equilibria have the same stability properties and undergo the same bifurcations.

Since $\left.1-T r+\operatorname{det}=2 \varepsilon\left(\mu^{2}-1\right)-(\mu+1)^{2}-1\right)>0$ for $\varepsilon>\varepsilon_{P}$, i.e., whenever $E_{1}$ and $E_{2}$ exist, the conditions for the eigenvalues to be inside the unit circle are det $<1$ and $1+T r+\operatorname{det}>0$ (see e.g., Gumowski and Mira, 1980, p. 159, or Medio and Lines, 2001, p. 52). The condition Det $<1$ becomes

$$
\varepsilon<\varepsilon_{H} \equiv \frac{1+\mu(\mu+2)}{2\left(\mu^{2}-2\right)}
$$

and the condition $1+\operatorname{Tr}+\operatorname{det}>0$ becomes

$$
\varepsilon<\varepsilon_{F E_{i}} \equiv \frac{\mu^{2}+2 \mu-3}{2\left(\mu^{2}-3\right)} .
$$

These two conditions define the stability region represented in Fig. 2(b). Notice that $\varepsilon_{F E_{i}} \leq \varepsilon_{H}$ for $\mu \geq(1+\sqrt{28}) / 3$ and $\varepsilon_{F E_{i}} \geq \varepsilon_{P}$ for $\mu \geq 3$. The last inequality 
confirms that the two fixed points $E_{i}, i=1,2$, are stable just above the curve $\varepsilon=\varepsilon_{P}$ (i.e., just after their creation) if $\mu>3$ (and consequently $\varepsilon<1$ ).

\section{Appendix B. Noninvertible Maps, Critical Curves and Non-Connected Basins}

In this appendix we give some basic definitions, properties and a minimal vocabulary of the theory of noninvertible maps of the plane and provide the reader with some basic facts about the method of critical curves. We also describe some properties of the critical curves of the map $\tilde{T}_{\lambda}$ defined in (45), from which also the analogous properties of the map $\tilde{T}$ given in (29) can be deduced as $\tilde{T}$ represents a particular case of $\tilde{T}_{\lambda}$ obtained for $\lambda=1$.

A two-dimensional map $T:(x, y) \rightarrow\left(x^{\prime}, y^{\prime}\right)$ transforms a point $(x, y) \in \mathbb{R}^{2}$ into a unique point $\left(x^{\prime}, y^{\prime}\right) \in \mathbb{R}^{2}$ according to the transformation

$$
\left(x^{\prime}, y^{\prime}\right)=T(x, y)=(f(x, y), g(x, y))
$$

where $(x, y) \in \mathbb{R}^{2}$ and $f, g$ are assumed to be real valued continuous functions. The point $\left(x^{\prime}, y^{\prime}\right) \in \mathbb{R}^{2}$ is called a rank-1 image of the point $(x, y)$ under $T$, and $(x, y)$ is called a rank-1 preimage of $\left(x^{\prime}, y^{\prime}\right)$. The point $(x(t), y(t))=T^{t}(x, y), t \in \mathbb{N}$, is called an image (or forward iterate) of rank- $t$ of the point $(x, y)$, where $T^{0}$ is identified with the identity map and $T^{t}(\cdot)=T\left(T^{t-1}(\cdot)\right)$. If distinct points, say $\left(x_{1}, y_{1}\right) \neq\left(x_{2}, y_{2}\right)$, exist that are mapped by $T$ into the same point, say $\left(x^{\prime}, y^{\prime}\right)=T\left(x_{1}, y_{1}\right)=T\left(x_{2}, y_{2}\right)$, then the map $T$ is said to be a noninvertible map. This can be equivalently stated by saying that the point $\left(x^{\prime}, y^{\prime}\right)$ has several rank-1 preimages, denoted by $T_{j}^{-1}\left(x^{\prime}, y^{\prime}\right)$, $j=1, \ldots, k$. This notation can also be expressed by saying that the inverse relation $T^{-1}$ is a multivalued function. A noninvertible map has the geometric property of "folding" the phase space, so that distinct points are mapped into the same point. Equivalently, the inverses are said to "unfold" the phase space.

As the point $\left(x^{\prime}, y^{\prime}\right)$ varies in the plane $\mathbb{R}^{2}$ the number of its rank-1 preimages can change. According to the number of distinct rank-1 preimages associated with each point of $\mathbb{R}^{2}$, the plane can be subdivided into regions, denoted by $Z_{k}$, whose points have $k$ distinct preimages. Generally, pairs of real preimages appear or disappear as the point $\left(x^{\prime}, y^{\prime}\right)$ crosses the boundary separating regions characterized by a different number of rank-1 preimages. Accordingly, such boundaries are generally characterized by the presence of two coincident (merging) preimages. This leads us to the definition of critical curves, one of the distinguishing features of noninvertible maps. The critical curve of rank-1, denoted by $L C$ (from the French "Ligne Critique") is defined as the locus of points having two, or more, coincident rank-1 preimages. These preimages are located in a set called the critical curve of rank-0, denoted by $L C_{-1}$. The curve $L C$ is the two-dimensional generalization of the notion of critical value (local minimum or maximum value) of a one-dimensional map, and $L C_{-1}$ is the generalization of the notion of critical point (local extremum point). As in the case of differentiable one-dimensional maps, where the derivative necessarily vanishes at the local extremum points, for a two-dimensional continuously 
differentiable map the set $L C_{-1}$ belongs to the set of points in which the Jacobian determinant vanishes:

$$
L C_{-1} \subseteq\left\{(x, y) \in \mathbb{R}^{2} \mid \operatorname{det} D T=0\right\} .
$$

In fact, since $L C_{-1}$ is defined as the locus of coincident rank-1 preimages of the points of $L C$, in any neighborhood of a point of $L C_{-1}$ there are at least two distinct points mapped by $T$ in the same point near $L C$. This means that the map $T$ is not locally invertible in the points of $L C_{-1}$ and, if the map $T$ is continuously differentiable, it follows that $\operatorname{det} D T$ necessarily vanishes along $L C_{-1}$. If the set $L C_{-1}$ is determined by (B.2) then $L C$ is simply obtained as the image of $L C_{-1}$, i.e., $L C=T\left(L C_{-1}\right)$.

In order to give a geometrical interpretation of the "unfolding action" of the multivalued inverse relation $T^{-1}$, it is useful to consider a region $Z_{k}$ as the superposition of $k$ sheets, each associated with a different inverse. Such a representation is known as Riemann foliation of the plane (see e.g., Mira et al., 1996a, b). Different sheets are connected by folds joining two sheets, and the projections of such folds on the phase plane are $\operatorname{arcs}$ of $L C$.

The map $\tilde{T}_{\lambda}$ defined in Eq. (45) is a noninvertible map. In fact, given a point $\left(q_{1}^{\prime}, q_{2}^{\prime}\right)$, its preimages are computed by solving the following system with respect to $q_{1}$ and $q_{2}$ :

$$
\left\{\begin{array}{l}
\left(1-\lambda_{1}\right) q_{1}+\lambda_{1} \mu_{1}\left[\varepsilon_{1} q_{2}\left(1-q_{2}\right)+\left(\varepsilon_{1}-1\right) q_{1}\left(1-\frac{\varepsilon_{1}-1}{\varepsilon_{1}} q_{1}-2 q_{2}\right)\right]=q_{1}^{\prime} \\
\left(1-\lambda_{2}\right) q_{2}+\lambda_{2} \mu_{2}\left[\varepsilon_{2} q_{1}\left(1-q_{1}\right)+\left(\varepsilon_{2}-1\right) q_{2}\left(1-\frac{\varepsilon_{2}-1}{\varepsilon_{2}} q_{2}-2 q_{1}\right)\right]=q_{2}^{\prime} .
\end{array}\right.
$$

This is a fourth degree algebraic system, which may have four or two real solutions or no real solution at all. Following the terminology of Mira et al. (1996a), we say that the map $\tilde{T}_{\lambda}$ is a noninvertible map of $Z_{4}-Z_{2}-Z_{0}$ type. Moreover, for the map $\tilde{T}_{\lambda}$ the set $L C_{-1}$ coincides with the set of points at which $\operatorname{det} D \tilde{T}_{\lambda}=0$, where

$$
\begin{aligned}
& D \tilde{T}\left(q_{1}, q_{2}\right) \\
& \quad=\left[\begin{array}{cc}
1-\lambda_{1}+\lambda_{1}\left(\varepsilon_{1}-1\right) \mu_{1}\left(1-2 \frac{\varepsilon_{1}-1}{\varepsilon_{1}} q_{1}-2 q_{2}\right) & \lambda_{1} \mu_{1}\left[\varepsilon_{1}\left(1-2 q_{2}\right)-2\left(\varepsilon_{1}-1\right) q_{1}\right] \\
\lambda_{2} \mu_{2}\left[\varepsilon_{2}\left(1-2 q_{1}\right)-2\left(\varepsilon_{2}-1\right) q_{2}\right] & 1-\lambda_{2}+\lambda_{2}\left(\varepsilon_{2}-1\right) \mu_{2}\left(1-2 \frac{\varepsilon_{2}-1}{\varepsilon_{2}} q_{2}-2 q_{1}\right)
\end{array}\right],
\end{aligned}
$$

so that the locus of points at which $D \tilde{T}\left(q_{1}, q_{2}\right)=0$ is a second order algebraic curve, which represents a hyperbola. So, $L C_{-1}$ is formed by the union of two disjoint branches, say $L C_{-1}=L C_{-1}^{(a)} \cup L C_{-1}^{(b)}$, see Fig. 7. Also $L C=T\left(L C_{-1}\right)$ is the union of two branches: $L C^{(a)}=T\left(L C_{-1}^{(a)}\right)$ and $L C^{(b)}=T\left(L C_{-1}^{(b)}\right)$. The branch $L C^{(a)}$ separates the region $Z_{0}$, whose points have no preimages, from the region $Z_{2}$, whose points have two distinct rank-1 preimages. The other branch $L C^{(b)}$ separates the region $Z_{2}$ from $Z_{4}$, whose points have four distinct preimages (see again Fig. 7 ).

This allows us to explain the contact bifurcation that causes the transformation of the connected basin $\mathcal{B}\left(E_{2}\right)$ shown in Fig. $7(\mathrm{a})$ into the non-connected basin $\mathcal{B}\left(E_{2}\right)$ 
shown in Fig. 7(b). In fact, in Fig. $7(\mathrm{a}), W^{s}(S)$, i.e., the boundary $W^{s}\left(E_{S}\right)$ which separates the two basins, is entirely included inside the regions $Z_{2}$ and $Z_{0}$. However, the fact that in Fig. $7\left(\right.$ a) a portion of it is close to the critical curve $L C^{(b)}$ suggests that if some of the parameters are changed, so that a contact between $W^{s}(S)$ and $L C$ occurs, this contact will mark a bifurcation which causes qualitative changes in the structure of the basins. In fact, if a portion of $\mathcal{B}\left(E_{2}\right)$ enters $Z_{4}$ after a contact with $L C^{(b)}$ has occurred, new rank-1 preimages of that portion will appear near $L C_{-1}^{(b)}$, and such preimages must belong to $\mathcal{B}\left(E_{2}\right)$. This is the situation shown in Fig. 7 (b) which is obtained by slightly changing the inertia parameter $\lambda_{2}$. The portion of $\mathcal{B}\left(E_{2}\right)$ inside $Z_{4}$, denoted by $H_{0}$ in Fig. $7(\mathrm{~b})$, has two rank-1 preimages, denoted by $H_{-1}^{(1)}$ and $H_{-1}^{(2)}$, which are located at opposite sides with respect to $L C_{-1}^{(b)}$ and merge on it (in fact, by definition, the rank-1 preimages of the arc of $L C^{(b)}$ which bound $H_{0}$ must merge along $\left.L C_{-1}^{(b)}\right)$. The set $H_{-1}=H_{-1}^{(1)} \cup H_{-1}^{(2)}$ constitutes a non connected portion of $\mathcal{B}\left(E_{2}\right)$. Moreover, since $H_{-1}$ belongs to the region $Z_{4}$, it has four rank-1 preimages, denoted by $H_{-2}^{(j)}, j=1, \ldots, 4$ in Fig. $7($ b), which constitute four other "islands" of $\mathcal{B}\left(E_{2}\right)$. Points of these "islands" are mapped into $H_{0}$ after two iterations of the map $\tilde{T}_{\lambda}$. Hence, at the contact between $W^{s}(S)$ and $L C$, the basin $\mathcal{B}\left(E_{2}\right)$ is transformed from a simply connected into a non connected set: the larger connected component of $\mathcal{B}\left(E_{2}\right)$, which contains $E_{2}$, is called the immediate basin $\mathcal{B}_{0}\left(E_{2}\right)$, and the whole basin is given by the union of its preimages: $\mathcal{B}\left(E_{2}\right)=\bigcup_{k \geq 0} T^{-k}\left(\mathcal{B}_{0}\left(E_{2}\right)\right)$. Notice that the exact values of the parameter at which such contact bifurcations occur cannot be computed analytically, as we do not have an equation for the boundary $W^{s}\left(E_{S}\right)$. However, the occurrence of this bifurcation can be detected by computer-assisted proofs, based on the knowledge of the properties of the critical curves and their graphical representation (see e.g., Mira et al., 1996a, for many examples). This "modus operandi" is typical in the study of the global bifurcations of nonlinear two-dimensional maps.

We end this Appendix by stressing that if both $\varepsilon_{i}=1$, then the locus of the vanishing Jacobian is given by the equilateral hyperbola of equation

$$
\left(q_{1}-\frac{1}{2}\right)\left(q_{2}-\frac{1}{2}\right)=\frac{\left(1-\lambda_{1}\right)\left(1-\lambda_{2}\right)}{4 \lambda_{1} \lambda_{2} \mu_{1} \mu_{2}}
$$

and if $\lambda_{1}=1$ or $\lambda_{1}=1$ this degenerates into the union of the two lines of equation $q_{1}=1 / 2$ and $q_{2}=1 / 2$. The case with $\varepsilon_{1}=\varepsilon_{2}=1$ and $\lambda_{1}=\lambda_{2}=1$ corresponds to the Cournot tâtonnement studied in Bischi et al. (2000), where it is shown that the property of having $L C_{-1}$ formed by vertical and horizontal lines, together with the properties that any map of the form (26) maps vertical lines into horizontal lines and vice-versa, gives rise to particular global properties of the chaotic areas and the basin boundaries, namely that the chaotic areas, as well as their basins, are characterized by rectangular boundaries. 


\section{References}

Abraham, R., Gardini, L. and Mira, C. [1997] Chaos in Discrete Dynamical Systems (A Visual Introduction in Two Dimensions) (Springer-Verlag).

Bischi, G. I., Mammana, C. and Gardini, L. [2000] "Multistability and cyclic attractors in duopoly games," Chaos, Solitons and Fractals 11, 543-564.

Bischi, G. I. and Kopel, M. [2001] "Equilibrium selection in a nonlinear duopoly game with adaptive expectations," Journal of Economic Behavior and Organization 46(1), $73-100$.

Bischi, G.-I., Gallegati, M. and Naimzada, A. [1999] "Symmetry-breaking bifurcations and representative firm in dynamic duopoly games," Annals of Operations Research $\mathbf{8 9}$, $253-272$.

Brousseau, V. and Kirman, A. [1992] "Apparent convergence of learning processes in mis-specified games," in Game Theory and Economic Applications, eds. Dutta, B., Mookherjee, D., Partasarathy, T., Raghavan, T. E. S., Ray, D. and Tijs, S. (Springer), 303-331.

Brousseau, V. and Kirman, A. [1993] "The dynamics of learning in N-person games with the wrong N," in Frontiers of Game Theory, eds. Binmore, K., Kirman, A. and Tani, P. (MIT Press), 71-93.

Bulow, J. I., Geanakoplos, J. D. and Klemperer, P. D. [1985a] "Multimarket oligopoly: Strategic substitutes and complements," Journal of Political Economy 93, 488-511.

Bulow, J. I., Geanakoplos, J. D. and Klemperer, P. D. [1985b] "Holding the capacity to deter entry," The Economic Journal 95, 178-182.

Canovas, J. S. [2000] "Chaos in duopoly games," Nonlinear Studies 1(1), 97-104.

Canovas, J. S. and Linero, A. [2001] "Topological dynamic classification of duopoly games," Chaos, Solitons \& Fractals 1(12), 1259-1266.

Chiarella, C. and Szidarovszky, F. [2001] "The nonlinear cournot model under uncertainty with continuously distributed time lags," Central European Journal of Operations Research 9, 183-196.

Chiarella, C., Szidarovszky, F. and Zhu, P. [2002] "The interaction of uncertainty and information lags in the cournot oligopoly model," in Oligopoly Dynamics; Models and Tools, eds. Puu, T. and Sushko, I. (Springer-Verlag), 233-263.

Dana, R. A. and Montrucchio, L. [1986] "Dynamic complexity in duopoly games," Journal of Economic Theory 40, 40-56.

Fudenberg, D. and Levine, D. [1998] The Theory of Learning in Games (MIT Press).

Gates, D. J., Rickard, J. A. and Wilson, D. J. [1978] "Convergence of a related game strategy," Journal of Mathematical Economics 5, 97-110.

Gates, D. J., Rickard, J. A. and Wilson, D. J. [1982] "Exact cooperative solution of a duopoly model without cooperation," Journal of Mathematical Economics 9, 27-35.

Gumowski, I. and Mira, C. [1980] Dynamique Chaotique, Cepadues editions (Toulose).

Huang, W. [2001] "Theory of adaptive adjustment," Discrete Dynamics in Nature and Society 5, 247-263.

Huck, S., Normann, H.-T. and Oechssler, J. [1999] "Learning in cournot oligopoly — an experiment," The Economic Journal 109(454), C80-C95.

Kirman, A. P. [1975] "Learning by firms about demand conditions," in Adaptive Economic Models, eds. Day, R. H. and Groves, T. (Academic Press), 137-156.

Kirman, A. P. [1983] "On mistaken beliefs and resultant equilibria," in Individual Forecasting and Aggregate Outcomes, eds. Frydman, R. and Phelps, E. S. (Cambridge University Press), 147-166. 
Kirman, A. P. [1995]) "Learning in oligopoly: Theory, simulation, and experimental evidence," in Learning and Rationality in Economics, eds. Kirman, A. and Salmon, M. (Blackwell), 127-178.

Kirman, A. P. and Salmon, M. (eds.) [1995] Learning and Rationality in Economics (Blackwell).

Kopel, M. [1996] "Simple and complex adjustment dynamics in cournot duopoly models," Chaos, Solitons 83 Fractals 7(12), 2031-2048.

Kopel, M., Bischi, G.-I. and Gardini, L. [2000] "On new phenomena in dynamic promotional competition models with homogeneous and quasi-homogeneous firms," in Interaction and Market Structure. Essays on Heterogeneity in Economics, eds. Delli Gatti, D., Gallegati, M. and Kirman, A. P. (Springer-Verlag), 57-87.

Léonard, D. and Nishimura, K. [1999] "Nonlinear dynamics in the Cournot model without full information," Annals of Operations Research 89, 165-173.

Marimon, R. [1997] "Learning from learning in economics," in Advances in Economics and Econometrics, eds. Kreps, D. and Wallis, K. (Cambridge University Press).

Medio, A. and Lines, M. [2001] Nonlinear Dynamics (Cambridge University Press).

Mira, C., Gardini, L., Barugola, A. and Cathala, J. C. [1996a] Chaotic Dynamics in TwoDimensional Noninvertible Maps (World Scientific, Singapore).

Mira, C., Carcasses, J. P., Gilles, M. and Gardini, L. [1996b] "Plane foliation of two dimensional noninvertible maps," International Journal of Bifurcation and Chaos 6(8), 1439-1462.

Poston, T. and Stewart, I. [1978] Catastrophe Theory and its Applications (Pitman).

Puu, T. [1991] "Chaos in duopoly pricing," Chaos, Solitons \& Fractals 1(6), 573-581.

Rand, D. [1978] "Exotic phenomena in games and duopoly models," Journal of Mathematical Economics 5, 173-184.

Rassenti, S., Reynolds, S. S., Smith, V. L. and Szidarovszky, F. [2000] "Adaptation and convergence in repeated experimental cournot games," Journal of Economic Behavior and Organization 41, 117-146.

Schaffer, S. [1984] "Chaos, naivete, and consistent conjectures," Economics Letters 14, $155-162$.

Schinkel, M. P., Tuinstra, J. and Vermeulen, D. [2002] "Convergence of Bayesian learning to general equilibrium in mis-specified models," Journal of Mathematical Economics 38, 483-508.

Szidarovszky, F. and Krawczyk, J. [2003] "On stable learning in dynamic oligopolies," Working paper, Department of Industrial Engineerig, University of Arizona.

Van Huyck, J. B., Cook, J. P. and Battalio, R. C. [1994] "Selection dynamics, asymptotic stability, and adaptive behavior," Journal of Political Economy 102, 975-1005.

Van Witteloostuijn, A. and Van Lier, A. [1990] "Chaotic patterns in Cournot competition," Metroeconomica 41, 161-185.

Wenzelburger, J. [2003] "Learning to play best response," forthcoming in International Game Theory Review. 